\title{
The Interpretation of Early-Type Spectra
}

\author{
A. B. UNDERHILL
}

Sonnenborgh Observatory, University of Utrecht, The Netherlands

\begin{abstract}
SUMMARX
In this paper the present position concerning the meaning of spectral type among the 0 - and B-stars in terms of physical conditions in the stellar atmosphere and the abundances of the elements is reviewed. In Section 2 the question "what is a spectral type" is examined and it is noted that because absorptionline spectral types and photometrical spectral types are based on dufferent criteria, the one is not necessarly always consistent with the other. The slope of the Paschen contunuum appears to be a good index of effective temperature at types $\mathrm{B} 2$ and later, while the wings of $H \gamma$ give a consistent means of determining $\log g$. In Section 3 the meaning of spectral type is exammed from a theoretical viewpoint. First the simple physical concepts underlyng present theories for constructing model atmospheres and predicting theoretical spectra are reviewed and then the results obtaned from a series of model atmospheres representing, in principle, main.sequence stars of types B6 to 09 are compared with observed details in stellar spectra.

It is shown that the adopted simple theory of spectrum formation, which is based on the concept of local thermodynamic equilibrium, is completely inadequate for understanding the formation and strengths of the strong lines which are used to define spectral types Bl to O5, and even among the stars of type B2 and later the agreement between predicted and observed spectra leaves much to be desired. Examples are presented to demonstrate the difficulty of interpreting line shapes in a unique manner. In Section 4 it 18 shown that the electron density in the parts of the atmosphere where the classification lines are formed is low enough that considerable doubt exists about the validity of the concept local thermo. dynamic equilibrium for many key lines and a summary is given of the observational evidence indicating that many of the dommant characteristics of 0 - and B-type spectra are due to particular processes of excitation and ionization.
\end{abstract}

\section{INTRODdCTION}

Intuitively one realizes that the detailed intensity distribution of the light emerging from stellar atmospheres must carry information concerning the physical conditions in the layers of the atmosphere from which the light escapes. In this paper an attempt is made to summarize and assess the knowledge that has been obtained about conditions in the atmospheres of stars of types A 5 and earlier-the early-type stars. Information is sought about the chemical composition of the atmosphere and its physical state. In particular one would like to be able to estimate the effective temperature and radius of a star from the appearance of the spectrum or from narrow-band photometric measurements in order to place the star more or less exactly in one or other theoretical scheme of evolution. 
The brighter stars have been sorted into groups according to empirically selected criteria representing the spectral dustribution in the range 3900-5000 A. A spectral type has been assigned to each class which can be distinguished, and these types have been arranged in a sequence according to increasing level of excitation. The theoretical studies to be reviewed have been aimed at obtaining an understanding of the meaning of the spectral sequence. If one supposes that the chemical composition of all stellar atmospheres is the same (this seems to be a reasonable hypothesis considering the great similarities between most stellar spectra) and that the atmosphere may be treated as a gas in thermodynamic equilibrium through which radiation is flowing, the spectral sequence can be understood, by and large, as a result of an increasing temperature in the atmosphere as one goes from type A through type B to type 0 However, such simple hypotheses do not give a completely satisfactory interpretation of the details visible in early type spectra. One purpose of this paper is to show what types of discrepancy occur and to indicate where more exact ways are needed of representing the physical interaction between gas and radiation which results in a stellar spectrum

The method used is direct Make a model of a stellar atmosphere and predict in detal the part of the spectrum which is observed Compare the predicted continuous spectrum and line spectrum with quantitative measurements of observed spectra. Vary the free parameters of the model untıl coincidence with the observed spectrum is obtained. In this way one can, in principle, determine the relative abundances of the elements and an approximation to the temperature and pressure structure of the atmosphere.

The answers which are obtained-temperatures, electron densities, state of motion, relative abundances-are not independent of the hypotheses made for constructing the model nor of the theory of spectrum formation which is used Since it has been impractible to use very detailed models and theories and since the available observational data are few in number and not always so precsse as required to differentiate between different theoretical representations, the resultant interpretations of stellar spectra are usually only best guesses. It is important to decide upon the true meaning of the remaining discrepancies between theory and observation, for the results of spectral analysis are used together with theories of stellar evolution to deduce certain characteristics of the Universe and how the Universe was formed and is evolving.

\section{What is a Spectral Type?}

Since the stars to be interpreted are selected according to their spectral type it is necessary to note what a spectral type is. There are, indeed, different definitions which are not entirely compatible, the one with the other. The incompatibilities arise because different criteria are used.

\section{A. Absorption-line Spectral Types}

Originally spectral types were assigned according to empirically selected criteria regarding the relative intensities of a few strong absorption lines in the part of the spectrum lying between 3900 and $5000 \AA$. If emission lines appear, the spectrum is noted as peculiar and placed in a particular class. Since the classifications were performed in the case of the Henry Draper Catalogue (Cannon and Pickering, 1918) and similar compilations using rather low-dispersion, low spectral purity spectra, it was necessary to select rather strong lines as the spectral-type criteria. Classifications of early type stars based on objectiveprism spectra are generally fairly consistent, but they indicate only the most conspicuous trends shown by the strongest spectral lines. 
When slit spectra of somewhat higher dispersion and of well-defined spectral purity (defined by the slit width, emulsion type, and method of development) are used, it is possible to classify stellar spectra more consistently and in greater detail. This increase in precision is reached by careful prescription of the quality of the spectrograms and by using criteria which involve some of the weaker lines in the spectrum. In particular it has been possible to develop two-dimensional systems giving spectral class and luminosity. In the MK classification system (Morgan and Keenan, 1951; Morgan and Johnson, 1953), the spectral type is defined by the apparent relative intensity of selected absorption lines while the luminosity class is given by other line ratios. Among the stars of type A5 and earlier the apparent shape of $H \gamma$ and $H \delta$ is an important luminosity criterion.

The classification system for B and $O$ stars developed at the Dominion Astrophysical Observatory (Plaskett and Pearce, 1930, Petrie, 1964) is similar in many respects, the apparent relative strengths of the stronger absorption lines being used to assign the spectral type while the luminosity is found from the measured equivalent width of $H \gamma$. In both classification systems, the spectral type is a notation which summarizes the relative strengths of a few absorption lines in the spectrum.

The spectral types lying between classes A5 and B5 are difficult to assign because most of the spectral lines except those due to hydrogen are rather weak. There are very few lines of intermediate strength which can be used effectively to distinguish the various sub-classes. With well-widened spectra of moderate dispersion $(30-50 \AA / \mathrm{mm})$ it is possible to detect the weaker lines. It is then found that there is a considerable variety in detail. In a few stars lines of Si II are relatively prominent to what they are in the vast majority of stars having roughly similar spectra; in other stars lines of $\mathrm{Mn} \mathrm{II}$ or $\mathrm{Cr}$ II and Eu II predominate, and so forth. These irregularities in detail have led to the recognition of "peculiar" classes, designated Bp or Ap. Among the spectral types of somewhat lower excitation one obtains the Am stars. These are the "metallic-line" stars. In this case the absorption lines due to the first and second spectra of the metals are stronger than would be expected according to the norm established by the majority of stars having hydrogen lines of similar strengths. In the case of the Bp, Ap and Am stars, the standard spectraltype criteria are not sufficient to permit a single-valued prediction of the rest of the spectrum. For a full description the absence or presence of other lines must be considered.

The fact that the empirically selected spectral type and luminosity criteria do not always permit one to isolate stars having nearly identical spectra raises the question of what are good criteria which will enable us to determine uniquely the effective temperature and radius of the star from the appearance of its spectrum. Such criteria are needed if one is to relate observed HR diagrams uniquely with the equivalent diagrams derived from theories of stellar evolution.

\section{B. Photometric Spectral Types}

With the need to survey and classify many faint stars in connection with problems of stellar evolution, attention has turned to assigning spectral types according to the values of photometric criteria. This is done by means of empirical relationships between broad or narrow band colour indices, gradients, or measures of the magnitude of the Balmer jump and the empirically defined absorption-line spectral types. Thus one has relationships of the sort

$$
\begin{aligned}
(U-B)_{0} \quad \text { or } \quad & (B-V)_{0} \text { vs. spectral type, } \\
& (b-v)_{0} \text { vs. spectral type, and } \\
& \Phi_{b}, D \quad \text { vs. spectral type. }
\end{aligned}
$$


In the first three relations the luminosity classification of the star in the case of $B$ stars is performed by measuring photometrically the strength of the $H \beta$ absorption line.

Another development has been to attempt to use model atmospheres to establish durect relationships between effective temperature and gradients measured either by well-known photometric colour indices or from low resolution spectral scans. In this case the stars studied are restricted to main-sequence stars, the luminosity classification being made from the empirically established spectroscopic luminosity criteria.

The type of result obtained is shown in Fig. 1 which is due to Heintze (1969). Here the stars are plotted according to the spectral type assigned from inspecting the spectrum and according to the effective temperature resulting from correlations which have been established, by means of model atmospheres, between photometric indices and effective

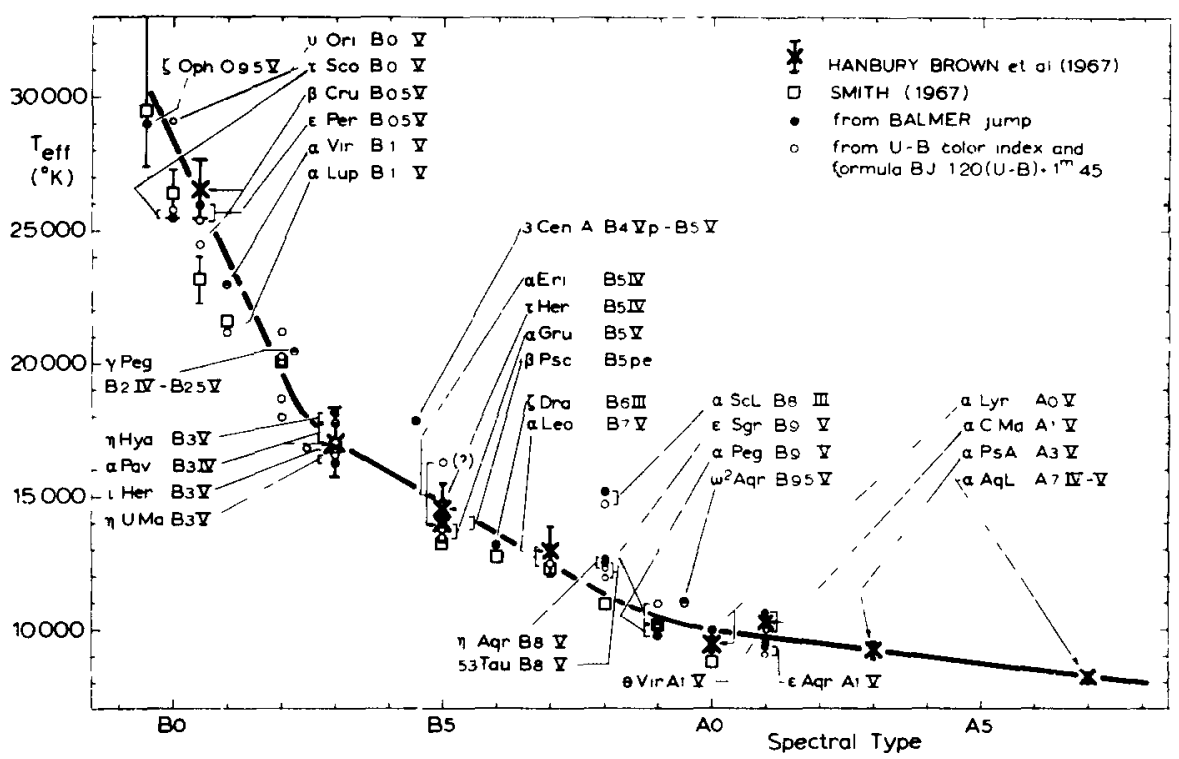

Fra. 1. The empirical effective temperature scale for the B stars due to Heintze.

temperature. The effective temperatures due to Hanbury Brown et al. (1967) have been found by relating the observed brightness temperature to effective temperature by means of a relation established using line-blanketed model atmospheres Those of Smith (1967) are from photometric measurements in the far ultraviolet Clearly there is a rather well defined relationship between spectroscopic spectral type and effective temperature through class B However, those stars which have peculiar spectra, in particular 3 Centauri $A$ and $\alpha$ Sculptoris, tend to fall rather far from the curve. If it is assumed that the effective temperatures determined from the photometric measurements are correct for these stars, then the assigned spectral types are inconsistent These discrepancies illustrate the fact that the empurical relationships used to establısh a spectroscopıc spectral type are not always consistent with the energy distribution in the continuous spectrum

At first it was thought that the relationships between photometric colour indices and spectral type were single-valued, but more detailed investigation has shown that among the B stars having a similar intrnsic colour stars with strong He I lines and stars with weak He I lines can occur (cf. Garrison, 1967, Bernacca, 1968). Among the A stars the same thmg is true the shape of the continuous spectrum in the usually observed spectral 
range may be the same for pairs of Ap or Am and normal A stars although the details of the line spectrum are different (cf. Sargent, 1964; Ledoux and Renson, 1966; Jugaku and Sargent, 1968). Further ambiguities are introduced in the interpretation of photometric spectral types by the fact that if a star rotates rapidly the total radiation flux emergent at the poles will differ from that emergent at the equator (Collins, 1966 ; Faulkner, Roxburgh, and Strittmatter, 1968). The measured radiation refers to an average radiation field from the star. The weighting function used in forming the average depends upon the inclination of the axis of rotation to the line of sight. In order to correct for the effects of rotation, one must have information about the projected rotational velocity of the star and of the orientation of the axis of rotation. Such information can be deduced in a few cases from the shapes of the lines and knowledge of the star's motion in a binary orbit.

\section{The Selection of Criteria for Effective Temperature and Radius}

By definition the effective temperature of a star represents the integrated radiation field

$$
\sigma_{R} T_{\text {eff }}^{4}=\pi \int_{0}^{\infty} F_{v}(0) d \nu,
$$

where $\sigma_{R}$ is Stefan's constant. In the case of early-type stars an important part of the radiation field emerges at wavelengths inaccessible from the surface of the earth and the integral of Eq. (1) cannot be evaluated directly unless adequate observational data corrected for interstellar reddening is available from instruments carried above the earth's atmosphere in rockets or satellites.

At present the most efficient method of finding the effective temperature of the early type stars is to predict for selected values of the constant $T_{\text {eff }}$ the detailed behaviour of the function $F_{v}(0)$ using the theory of model atmospheres and of spectrum formation and then to compare a few details in the part of the computed spectrum accessible from the surface of the earth with observed details in a stellar spectrum in order to identify a model with a star and in this way build up a relationship between $T_{\text {eff }}$ and spectral type. By good criteria for spectral type one means spectral details which are sensitive to variations in $T_{\text {eff }}$ but not to the other free parameters in the theoretical representation. If possible these criteria should be insensitive to the effects of interstellar reddening. Otherwise the observed effective temperature criteria must be derived from unreddened stars (almost unknown among early-type stars) or they must be corrected by assuming a wavelength dependence for the interstellar reddening law. This latter correction can be carried out with moderate confidence over ranges of $3000-4000 \AA$ centered at $5000 \AA$, so far as the relative distortion of the intensity of the starlight at different wavelengths is concerned. The observed shape of the reddening law is used, but considerable uncertainty still remains concerning the total absorption which occurs at any one wavelength.

In order to escape the difficulties with interstellar reddening one would like to look for absorption lines which can be used as criteria of effective temperature among the early type stars. However, as is shown below, the theoretical representation of the formation of the strong lines in early type stars is at present not satisfactory, thus preference returns to the use of the shape of the continuous spectrum as a criterion of effective temperature.

A good spectroscopic criterion for the effective temperature of early type stars is the shape of the continuous spectrum in regions above and below the Balmer jump. This is best determined from low resolution scans or from narrow band photometry. Heintze (1968, 1969) and Jugaku and Sargent (1968) have discussed the problems which are encountered in obtaining observed results which are correct on an absolute scale, as they 
must be for comparison with the predictions from model atmospheres. Heintze has come to the conclusions regarding the effective temperatures of the B stars shown in Fig. 1. He has checked his deductions by means of the photometric measurements of Smith (1967) which give a directly measured integration of the right side of Eq. (1), and by means of the effective temperatures deduced by Hanbury Brown et al. (1967) from measured radil and knowledge of the absolute fluxes of a few stars.

The radius which is required for linking spectroscopic observations of stars with the theoretical results of studies of stellar evolution is the radius of the photosphere, that is of the part of the stellar atmosphere which is opaque at an average wavelength in the continuous spectrum. It is best found for early type stars by determining the value of $g$ which gives predicted $H \gamma$ profiles like observed profiles. To find the radius one must then adopt a mass for the star.

\section{The Mraning of Spectral Type}

\section{A. The Theoretical Concepts used to Predict Spectra}

Models which are constructed to represent the atmospheres of stars consist of a perfect gas of constant composition through which the pressure and temperature changes and through which a flux of radiation flows. Most model atmospheres are made for the purpose of interpreting the spectra of main-sequence stars. The variation of pressure with depth is found by postulating that the atmosphere be in hydrostatic equilibrium under the acceleration of gravity $g$. For main-sequence stars $g$ is of the order of $10^{4}$. A term in the radiation pressure gradient is taken into account. Thus the temperature-pressure structure of a model is found by integrating the equation

$$
\frac{d p_{g}}{d \tau}=\frac{g}{x}-\frac{d p_{r}}{d \tau}
$$

Here depth in the atmosphere is measured in terms of a characteristic optical depth $\tau$, which is a folding of the geometrical depth $z$ in terms of a suitably chosen mean opacity coefficient $\varkappa$. Different definitions of $x$ have been given by Underhill (1962a), Mihalas (1965), Strom and Avrett (1965) to mention only a few authors who have constructed rather extensive grids of models. The radiation pressure gradient may be found at each depth $\tau$ in the model from the expression

$$
\frac{d p_{r}}{d \tau}=\frac{\pi}{c} \int_{0}^{\infty} \frac{\varkappa_{v}}{\varkappa} F_{v}(\tau) d \nu .
$$

Here $\varkappa_{v}$ is the total opacity at frequency $v$ due to continuous and line sources of opacity and $F_{v}(\tau)$ is the monochromatic flux at depth $\tau$. When a model calculation is begun an approximate expression is introduced for $d p_{r} / d \tau$ as a function of $\tau$ and after that in model $n+1$ of a series which is being integrated the radiation pressure gradient for model $n$ 1s used. The correct temperature law $T(\tau)$ is found by an iterative procedure based on the condition that radiative equilibrium should exist.

An approximate temperature law $T(\tau)$ is used to start the calculations and the temperature law is corrected by an iterative procedure until that temperature law is found which is consistent with the condition that radiative equilibrium exists. Suitable computing algorithms based on the discussion by Avrett and Krook (1963) have been published 
by Mihalas (1965) and by Strom and Avrett (1965). Other useful algorithm exist (cf. Pecker, 1965). Automatic iterative methods to find a temperature law which is consistent with the condition that radiative equilibrium exists are fairly efficient for models with $T_{\text {eff }} \leqq 20,000^{\circ} \mathrm{K}$. However, acceptable models corresponding to spectral types earlier than B2, $T_{\text {eff }}>20,000^{\circ} \mathrm{K}$, are difficult to obtain, particularly if the opacity due to line blanketing in the region 912-1500 $\AA$ is included. In these cases one can approach the desired condition of radiative equilibrium most efficiently by an empirical technique. That is, arbitrary changes are made in $T(\tau)$, and the desired model is approached by trial and error. The chief drawback to this technique is that one cannot specify $T_{\text {eff }}$ exactly a priori. However, this is not serious, because from the observational side the effective temperatures of stars of types B2 to 0 are not known exactly. What is known, approximately, is the temperature in the line-forming layers and the empirical method permits one to match the model to this piece of observational knowledge.

The detailed intensity distribution in the spectrum is usually predicted by solving the Milne-Eddington transfer equation numerically for $F_{v}(0)$. Thus the electrons are considered to scatter light isotropically and coherently while the part of the source function representing the re-emission of light absorbed in the continuous spectrum and in lines is given by Kirchhoff's law. The distribution of the atoms and ions over their possible excited states is calculated by means of the Saha and Boltzmann laws in order to be able to evaluate $x_{v}$ and $l_{v}$ per gram of star material from the adopted relative abundances. These assumptions mean that the interaction between radiation and matter in the stellar atmosphere is treated as if at each depth thermodynamic equilibrium existed in accordance with the local temperature. All of the theoretical results to be presented have been computed using these simple approximations. The advantage of this procedure is that it permits one to condense the problem of spectrum formation in a non-homogeneous atmosphere into a form that can be handled rather efficiently by a large computer.

The formulas to be used for evaluating the continuous absorption coefficient $x_{v}$ in models representative of early type atmospheres are well known and they are presented in most articles giving grids of models. The opacity is due chiefly to $\mathrm{H}_{1} \mathrm{H}^{-}, \mathrm{He}$, and $\mathrm{He}$. At cooler temperatures other sources of opacity may be mcluded as seems advisable. The shape of the line absorption coefficient is usually given by the Voigt function $H(a, v)$ where $a$ is the damping constant measured in units of the Doppler width and $v$ is the displacement in the line in units of the Doppler width, or, in the case of hydrogen lines, by a function representing the Stark broadening due to protons and to electrons. The effects of small-scale motions (microturbulence) on the line profiles may be explored by makıng the Doppler width greater than the value corresponding to the local temperature. The $f$-values and damping constants for the lines are obtained from theoretical studies or from laboratory experiments.

The predicted spectra obtained using the theoretical concepts listed above provide a well-defined norm with which to compare real stellar spectra. On the whole the predicted spectrum in the wavelength range accessible from the surface of the earth resembles observed spectra fairly well. However, some discrepancies exist. These discrepancies must be used to check the adequacy of the simple theory which is used and to determine the values of the free parameters which describe the physical state of the star.

The parameters describing the star are $T_{\text {eff }}, g$, and the relative abundances of the elements. Other parameters such as $f$-values and damping constants describe the interaction between atoms, ions, and radiation In principle they are known from studies done in the laboratory or from theory; in practice some uncertainties remain and each comparison between predicted and observed stellar spectra must be used to control the consistency of the adopted physical parameters as well as to determine abundances, $T_{\text {eff }}$ and $g$. 
The hypotheses of hydrostatic equilibrium and radiative equilibrium in the stellar atmosphere can be defended on general grounds in the case of main-sequence stars of early type The hypothesis of local thermodynamic equilibrium stands on less secure ground. From a computational point of view this hypothesis has great convenience for it permits one to use relatively sumple relationships which, since they represent equilibrium conditions, tell nothing of the individual processes which occur. However, it appears that many of the characteristic features of stellar spectra reflect the action of particular processes, and that the hypothesis of local thermodynamic equilibrium cannot be maintained in many cases. This point will be exammed further below. The answer to the question whether the distribution of atoms and ions over the possible energy levels may be calculated by equilibrium relationships or not depends entirely on the spacing of the energy levels, the values of the radiative and collision cross-sections, the average energy of the electrons, and the intensity distribution of the radiation field in which the atoms are bathed Consequently a separate investigation should be made for each species of atom. General statements such as "the state of local thermodynamic equilıbrium exists in this atmosphere but not in that" have no meaning Such statements must be related to a particular species of atom or ion.

\section{B. Theoretical Predictions vs. Observation at Type O9V}

In this section the predictions made from five model atmospheres of approximately type $09 \mathrm{~V}$ will be compared with each other and with observed details in the spectrum of 10 Lacertae, a standard $09 \mathrm{~V}$ star, in order to demonstrate how sensitive the predicted spectrum is to the details of the prediction process and to find out if the theoretical spectrum is indeed like that of a sharp-lined standard $09 \mathrm{~V}$ star. The theoretical material has been discussed in part by De Groot and Underhill (1964) and by Underhill and De Groot (1964), and in full by Underhill (1968a). A comprehensive list of identifications in the spectrum of 10 Lacertae and of equivalent widths has been given by Underhill and De Groot (1965).

Table 1. The Characteristics of Five O-Type Model Atmospheres

\begin{tabular}{c|c|c|c|l}
\hline $\begin{array}{c}\text { Model } \\
\text { name }\end{array}$ & $\begin{array}{c}T_{\text {eff }} \\
\left({ }^{\circ} \mathrm{K}\right)\end{array}$ & $\log g$ & Opaerty sources & \multicolumn{1}{|c}{ Remarks } \\
\hline 98 & 33947 & $4 \cdot 0$ & H, He I, He I, electrons & Coarse $\tau$ scale near $\tau=0$ \\
P7 & 33965 & $4 \cdot 0$ & H, He I, He Ir, electrons & Fine $\tau$ scale near $\tau=0$ \\
140 & 33411 & $3 \cdot 7$ & H, He I, He I, electrons & Coarse $\tau$ scale near $\tau=0$ \\
P5 & 33556 & $3 \cdot 7$ & H, He I, He II, electrons & Fine $\tau$ scale near $\tau=0$ \\
PPB40 & 32023 & $4 \cdot 0$ & H, He I, He II, electrons & Very fine $\tau$ scale near $\tau=0$ \\
& & & and line blanketing & \\
\hline
\end{tabular}

The five model atmospheres used are listed in Table 1. The models belong to three series. Those listed by a number only belong to the original series of unblanketed models in which the smallest interval in $\tau$ was 0.01 . The models with one $P$ in their name belong to a sumilar series in which the smallest interval in $\tau$ is 0.001 , while model PPB40 belongs to a series with even finer spacing $\mathrm{m} \tau(\mathbf{0 . 0 0 2})$ in which line blanketing $(\mathrm{B})$ is considered. The temperature law corresponding to radiative equllibrium was found by trial and error, each model being numbered in sequence. The listed numbers correspond to the models selected to have the best characteristics for the present purpose. In every case the departures from radiative equilibrium are less than 0.5 per cent (the limit of accuracy of the integration formulas used) in the parts of the atmosphere important for predicting the emergent 
spectrum. At effective temperatures higher than $20,000^{\circ} \mathrm{K}$ no temperature correction process converges well, thus one might as well use trial and error to find a suitable model. The listed effective temperatures correspond to the integrated emergent flux (without lines in the region $\lambda>1500 \AA$ ) from the model. The temperature at depth $\tau=0.1$ lies between $29,500^{\circ}$ and $30,500^{\circ}$ in all five models. Models P5 and P7 are very similar to models 140 and 98 at $\tau>0.1$; they are superior to models 140 and 98 for $\tau<0 \cdot 1$.

The electron pressure-temperature structure of the models is shown in Fig. 2. As expected from the smaller value of $\log g$ in model P5, the electron pressure at a given temperature

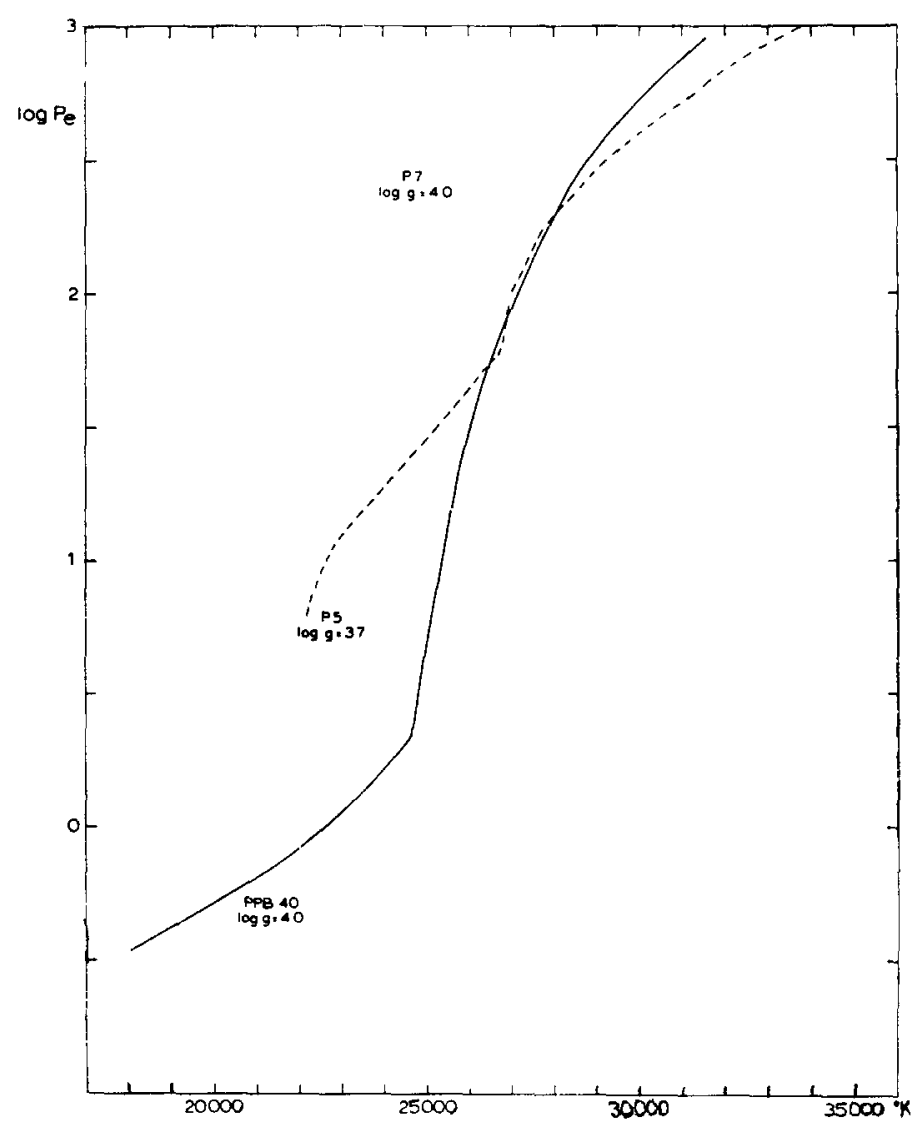

FIg 2. The electron pressure-temperature structure in three O-type model atmospheres.

is lower than in model P7. When line blanketing by the strong ultraviolet lines is also taken into account (model PPB40) the electron pressure is further lowered for a fixed value of $T$ in comparison to what it is in model $P 7$ which has the same value of $\log g$. At deeper layers in the model, not shown in the diagram, the pressure-temperature structure of PPB40 becomes more like that of P7. These results show that the pressure-temperature structure in the layers important for determining the emergent spectrum (according to the adopted simple theory of line formation) depends not only upon $\log g$ but also upon the adopted sources of opacity. Model PPB40 gives the most physically correct representation of an O-type atmosphere of the five models discussed here 
Details concerning the preducted continuous spectrum in the five models are given by Underhill (1968a). The relative intensity distribution in the continuous spectrum at $\lambda>2000 \AA$ is not particularly sensitive to the details of the model construction process, but the spectrum at shorter wavelengths is quite sensitive to the value of $\log g$ and to the sources of opacity. Although because of line blanketing, model PPB40 has a lower effective temperature than the other models, the brightness of the spectrum in the normally observed spectral region is a few per cent greater than it is for the unblanketed models. At wavelengths in the Lyman and He I continua model PPB40 is significantly brighter than the other models.

Line profiles have been preducted for many characteristic lines in O-type spectra using these five 0-type models and equivalent widths have been found. The results for model PPB40 in the case of the relative abundances listed in Table 2 are given in Table 3. Results for other cases can be found in the papers listed above. The adopted abundances are close to those recommended by Aller (1961) for the solar system. The fractional abundances by weight add up to a little more than unity This inconsistency occurs because the models are constructed of hydrogen and helium only while in order to compute lines small admixtures of the necessary elements are made.

Table 2. The Adopted Abundanges and the Most Probable Thermal Veloctty at $10,000^{\circ} \mathrm{K}$ and at $30,000^{\circ} \mathrm{K}$

\begin{tabular}{|c|c|c|c|c|c|c|}
\hline \multirow[t]{2}{*}{ Element } & \multirow{2}{*}{\multicolumn{2}{|c|}{$\begin{array}{l}\text { Fraotional } \\
\text { abundance } \\
\text { by welght }\end{array}$}} & \multirow{2}{*}{\multicolumn{2}{|c|}{$\frac{N \text { (element) }}{N(H)}$}} & \multicolumn{2}{|c|}{$\begin{array}{l}\text { Most probable thermal } \\
\text { velocity }\end{array}$} \\
\hline & & & & & $10,000^{\circ} \mathrm{K}$ & $30,000^{\circ} \mathrm{K}$ \\
\hline $\mathbf{H}$ & 0.68 & $0^{\mathrm{a}}$ & $1 \cdot 00$ & $0^{\mathrm{a}}$ & $12.85 \mathrm{~km} / \mathrm{sec}$ & $22 \cdot 3 \mathrm{~km} / \mathrm{sec}$ \\
\hline $\overrightarrow{\mathbf{H e}_{\mathbf{e}}}$ & 0.32 & 0 & 1.18 & -1 & $6.43 \mathrm{~km} / \mathrm{sec}$ & $11.1 \mathrm{~km} / \mathrm{sec}$ \\
\hline C & $3 \cdot 25$ & $-\mathbf{3}$ & $3 \cdot 99$ & -4 & $3.72 \mathrm{~km} / \mathrm{sec}$ & $6.44 \mathrm{~km} / \mathrm{sec}$ \\
\hline 0 & $9 \cdot 70$ & -3 & $8 \cdot 93$ & -4 & $3.21 \mathrm{~km} / \mathrm{sec}$ & $5.56 \mathrm{~km} / \mathrm{sec}$ \\
\hline $\mathrm{Ne}$ & $6 \cdot 89$ & $-\mathbf{3}$ & $5 \cdot 01$ & -4 & $2.86 \mathrm{~km} / \mathrm{sec}$ & $4.96 \mathrm{~km} / \mathrm{sec}$ \\
\hline $\mathrm{Mg}$ & $3 \cdot 28$ & -4 & 1.98 & -5 & $2.60 \mathrm{~km} / \mathrm{sec}$ & $4.51 \mathrm{~km} / \mathrm{sec}$ \\
\hline $\mathrm{S}_{1}$ & $1 \cdot 21$ & $-\mathbf{3}$ & $6 \cdot 33$ & -5 & $2.42 \mathrm{~km} / \mathrm{sec}$ & $4.20 \mathrm{~km} / \mathrm{sec}$ \\
\hline Ar & $2 \cdot 06$ & -4 & $7 \cdot 59$ & -6 & $2.03 \mathrm{~km} / \mathrm{sec}$ & $3.52 \mathrm{~km} / \mathrm{sec}$ \\
\hline $\mathrm{Ti}$ & $2 \cdot 53$ & -6 & $7 \cdot 80$ & -8 & $1.86 \mathrm{~km} / \mathrm{sec}$ & $3.22 \mathrm{~km} / \mathrm{sec}$ \\
\hline $\mathrm{Cr}$ & $8 \cdot 48$ & -6 & $2 \cdot 40$ & -7 & $1.78 \mathrm{~km} / \mathrm{sec}$ & $309 \mathrm{~km} / \mathrm{sec}$ \\
\hline $\mathrm{Fe}$ & 1.41 & -4 & $3 \cdot 7 \mathrm{I}$ & -6 & $1.72 \mathrm{~km} / \mathrm{sec}$ & $2.98 \mathrm{~km} / \mathrm{sec}$ \\
\hline
\end{tabular}

"The power of 10 by which to multiply the first number.

The hydrogen and He II profiles have been calculated using the $T(\beta, \gamma)$ Stark-broadening function of Griem (1960), see Underhill (1962b) for details. The revisions proposed by Griem later have little effect for $H \gamma$ and $H \delta$; the adopted Stark broadening function seriously overestimates the Stark broadening of $H \alpha$ and less seriously that of $H \beta$. The results for $H \alpha$ and $H \beta$ should be considered only to be numerical experiments made to find out what factors are significant for predicting these lines. The $T(\beta, \gamma)$ function has been folded with the Doppler broadening profile due to a Maxwell distribution of atom velocities with a most probable velocity $v_{p}$. The most probable thermal velocities at $10,000^{\circ} \mathrm{K}$ and $30,000^{\circ} \mathrm{K}$ for each atom or ion studied are listed in Table 2

The profiles of lines from other spectra than $\mathrm{H}$ and $\mathrm{He} \pi$ have been predicted using the Voigt broadening function $H(a, v)$. In each case the damping constant was assumed to be 10 times the classical radiation damping constant and all partition functions were put equal to the statistical weight of the ground term. Blended lines were computed when 
Table 3. Equivaleent Widthe and Cenntral Invmisities Predioted uging Model PPB40

\begin{tabular}{|c|c|c|c|c|c|c|c|c|}
\hline \multirow{2}{*}{\multicolumn{2}{|c|}{ Line }} & \multirow{2}{*}{$g f$} & \multicolumn{2}{|c|}{$\begin{array}{l}\text { Equivalent } \\
\text { width (A) }\end{array}$} & \multicolumn{2}{|c|}{$\begin{array}{l}\text { Central } \\
\text { Intensity }\end{array}$} & \multicolumn{2}{|c|}{$\begin{array}{l}\text { Observed } \\
10 \text { Lacertae }\end{array}$} \\
\hline & & & $1 \times \Delta v_{D}$ & $4 \times \Delta v_{D}$ & $1 \times \Delta v_{D}$ & $4 \times \Delta v_{D}$ & $W(A)$ & $\boldsymbol{R}$ \\
\hline \multicolumn{2}{|c|}{$\mathrm{H}+\mathrm{He} \pi \mathrm{H} \alpha$} & $5 \cdot 126$ & $2 \cdot 393$ & - & 0.451 & - & 3.916 & 0.572 \\
\hline \multicolumn{2}{|c|}{$\mathbf{H}+\mathbf{H e} \Pi \mathbf{H} \gamma$} & 0.955 & $1 \cdot 864$ & - & 0517 & - & $2 \cdot 893$ & 0.530 \\
\hline \multicolumn{2}{|c|}{$\mathbf{H}+\mathbf{H} \boldsymbol{\theta} \boldsymbol{I} \mathbf{H}$} & 0.357 & 1.927 & - & $0 \cdot 615$ & - & $2 \cdot 825$ & $0 \cdot 539$ \\
\hline \multicolumn{2}{|c|}{$\mathbf{H}+\overline{\mathbf{H e}} \mathbf{I} \mathbf{H} \boldsymbol{\delta}$} & 0.177 & 1.957 & - & 0.659 & - & $2 \cdot 568$ & $0 \cdot 556$ \\
\hline He II & 4686 & $15 \cdot 174$ & $1 \cdot 033$ & - & 0.596 & - & 0.997 & $0 \cdot 570$ \\
\hline He II & 5411 & $2 \cdot 096$ & 0.520 & - & $0 \cdot 829$ & - & $1 \cdot 34$ & 0.755 \\
\hline He II & 4541 & 0.598 & $0 \cdot 544$ & - & 0.888 & - & 1.087 & 0815 \\
\hline He II & 4200 & $0 \cdot 262$ & $0 \cdot 426$ & - & $0 \cdot 942$ & - & 0.523 & 0.872 \\
\hline $\mathrm{O} \pi$ & 4676 & 0.49 & 0.041 & $0 \cdot 062$ & 0.820 & 0.908 & 0.053 & 0.879 \\
\hline O II & 4661 & $0 \cdot 58$ & $0 \cdot 042$ & $0 \cdot 072$ & $0 \cdot 811$ & 0.896 & $0 \cdot 058$ & $0 \cdot 887$ \\
\hline $\mathbf{O} \mathbf{I I}+\mathbf{H}_{\gamma}$ & 4366 & 0.53 & $0 \cdot 037$ & 0073 & 0.803 & 0.895 & 0.074 & 0.867 \\
\hline $\mathrm{O} \Pi+\mathrm{H} \gamma$ & 4349 & 1.23 & 0.052 & 0.115 & 0.766 & 0.833 & 0.070 & 0.865 \\
\hline O III & 3444 & 0.55 & 0.030 & 0.059 & 0.822 & 0.898 & $0 \cdot 067$ & 0.914 \\
\hline O III & 3340 & $0 \cdot 45$ & 0.049 & 0.118 & 0.749 & $0 \cdot 815$ & $0 \cdot 188$ & 0.740 \\
\hline O III & 3312 & 0.27 & 0.044 & 0.096 & 0.769 & 0840 & $0 \cdot 115$ & 0.810 \\
\hline O III & 3299 & 0.09 & 0.027 & 0.052 & 0.822 & 0.904 & 0.058 & 0.873 \\
\hline Ne II & 3355 & $1 \cdot 22$ & 0.040 & 0.091 & 0.748 & 0.821 & 0.065 & 0.838 \\
\hline $\mathrm{Ne} I$ & 3344 & 0.48 & 0.081 & 0.048 & 0.793 & $0 \cdot 888$ & 0.073 & 0.912 \\
\hline $\mathrm{Ne}$ II & 3334 & $2 \cdot 33$ & 0.048 & 0.122 & 0.718 & 0.780 & $0 \cdot 149$ & 0.729 \\
\hline $\mathrm{Ne} I I$ & 3327 & 0.62 & 0032 & 0.058 & 0.778 & 0.867 & 0.061 & 0.862 \\
\hline $\mathrm{Ne} I I$ & 3311 & $0 \cdot 10$ & 0.011 & 0.014 & 0.900 & 0.969 & 0.040 & 0.882 \\
\hline $\mathrm{Ne} \Pi$ & 3297 & 053 & 0.032 & $0 \cdot 056$ & 0.782 & 0.876 & 0.039 & 0.906 \\
\hline $\mathbf{M g ~ I I}$ & $4481^{2}$ & - & $0 \cdot 034$ & $0 \cdot 034$ & $\rightarrow$ & 0.946 & 0.108 & 0.833 \\
\hline $\mathbf{S} \mathbf{I I}+\mathbf{H} \delta$ & $4130^{a}$ & $5 \cdot 94$ & - & - & - & - & 0.019 & 0.950 \\
\hline $\mathrm{Si} \mathbf{I I}+\mathbf{H} \boldsymbol{\delta}$ & $4128^{\circ}$ & $3 \cdot 96$ & - & - & - & - & 0.023 & 0937 \\
\hline Si III & 4552 & $2 \cdot 075$ & 0.027 & 0055 & 0.793 & 0.883 & 0.101 & 0.862 \\
\hline $\mathrm{Si} \mathbf{r v}+\mathbf{H} \delta$ & 4116 & 0.772 & 0.085 & 0.225 & $0 \cdot 656$ & 0.699 & $0 \cdot 229$ & $0 \cdot 687$ \\
\hline $\mathbf{S i} I V+\mathbf{H} \delta$ & 4088 & $1 \cdot 544$ & 0.108 & 0.244 & $0 \cdot 638$ & 0.675 & 0.305 & 0.606 \\
\hline Fe III & $4053 \cdot 1$ & $1 \cdot 204$ & 0.0007 & 0.0007 & 0.992 & 0.998 & 0.024 & 0.930 \\
\hline $\mathrm{Fe}$ III & $4137 \cdot 8$ & $4 \cdot 560$ & 0.0024 & $0 \cdot 0024$ & 0.973 & 0.992 & $0 \cdot 028$ & 0.938 \\
\hline Fe III & $4139 \cdot 3$ & $3 \cdot 534$ & 0.0017 & 0.0018 & 0.978 & 0.994 & $0 \cdot 015$ & 0.956 \\
\hline FeIII & $4140 \cdot 5$ & $1 \cdot 348$ & 0.0006 & 0.0007 & 0.991 & 0.998 & 0.011 & 0.960 \\
\hline Fe III & $4164 \cdot 7$ & $8 \cdot 846$ & 0.0037 & 00042 & 0.956 & 0.986 & 0.015 & 0.950 \\
\hline Fe III & 42386 & $0 \cdot 898$ & $0 \cdot 0004$ & $0 \cdot 0004$ & 0.995 & 0.999 & 0.015 & 0.952 \\
\hline
\end{tabular}

- See text.

necessary. The cases of blending are indicated in Table 3 by an obvious notation. The sources of the $g f$ values can be found by consulting the papers listed at the beginning of this section. The $g f$ value of $\mathrm{Mg} \Pi \mathbf{4 4 8 1 \cdot 1}$ is 5.70 while that of $\mathrm{Mg} \Pi \mathbf{4 4 8 1 \cdot 3}$ is $3 \cdot 80$. The central intensities of the two $\mathrm{Mg} \mathrm{II}$ lines are 0.889 and 0.914 , respectively, in the case of thermal Doppler broadening. With microturbulence they blend to form a line which does not appear to be double. The profiles of Si $\pi 4128$ and 4130 were computed in model 98 only and found to be extremely weak, having central absorptions of the order of 0.5 per cent.

The effect of microturbulence on the line profiles was investigated by introducing an arbitrary multiple of the Doppler halfwidth, $\Delta v_{D}$. Results are listed for 1 times, i.e. thermal Doppler broadening, and for 4 times $\Delta v_{D}$. Reference to Table 2 will show that for the light and heavy elements 4 times thermal Doppler broadening corresponds to a velocity distribution rather like that of the hydrogen atoms. Thus the "microturbulent velocities" which 
are used are not supersonic. The present numerical experiment to evaluate the effects of microturbulence corresponds to a velocity field which increases inwards. However, a modest range in the velocity dustribution which implies velocities increasing inwards or outwards in the atmosphere makes no significant change in the line profile (de Groot and Underhill, 1964). The chief factor is the suze of the average effective $\Delta v_{D}$ and not its variation with depth, so long as this variation is not great.

The observed equivalent widths and central depths in the spectrum of 10 Lacertae are given in Table 3 for the computed lines These two quantities serve to characterize the weaker lines in the spectrum. The stronger lines can best be studed by comparing observed and computed profiles. In the case of the stronger lines in the spectrum of 10 Lacertae the correction for the instrumental profile is negligible when spectrograms of $4.5 \AA / \mathrm{mm}$ or higher dispersion are studied since the stellar lines are not truly sharp, having total widths at half intensity of the order of $0 \cdot 5-0 \cdot 6 \AA$.

The observed profiles of $H \alpha, H \beta, H \gamma$, and $H \delta$ in 10 Lacertae are compared with some of the computed hydrogen line profiles in Fig. 3. It is clear that the observed hydrogen lines

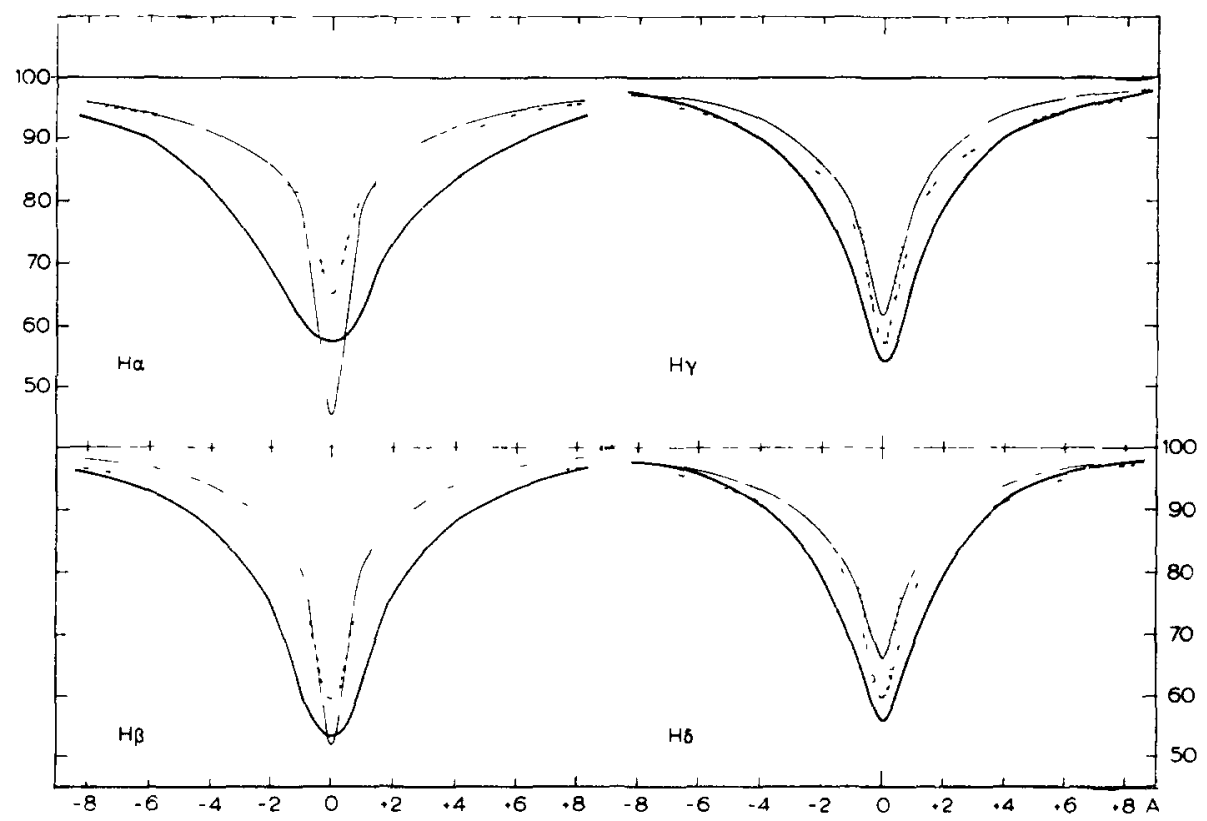

Fra. 3. The computed profiles of $H \alpha, H \beta, H \gamma$ and $H \delta$ in models P5, P7 and PPB40 compared with observed profiles in 10 Lacertae. The observed profiles are shown by a heavy line, those from model P5 by a dotted line, those from model P7 by a broken line and those from model PPB40 by a thin solid line.

in this standard $09 \mathrm{~V}$ star are not accurately represented by the adopted theory of line formation and the avalable models. The observed lines are significantly stronger than any of the computed profiles. A better fit in the cores might be obtained by introducing microturbulence, but the discrepancy is serious since the predicted cores using model PPB40, which because it includes blanketing by the ultraviolet lines and is close to radiative equilibrium appears in principle to be a good model, are significantly shallower for $H \gamma$ and $H \delta$ than observed The introduction of microturbulence will make the cores even shallower and broader. The discrepancies at the centres of the hydrogen lines are systematic. The 
observed hydrogen lines are rather like those from a model of later spectral type. The star $\mathrm{HD} 34078 \mathrm{AE}$ Aurigae is also classified as type 09V. Its $H \gamma$ profile is almost identical with that of 10 Lacertae. Thus these problems are not confined to 10 Lacertae. Scholz (1967) in his study of $\tau$ Scorpii also cannot match the $H \gamma$ profile well.

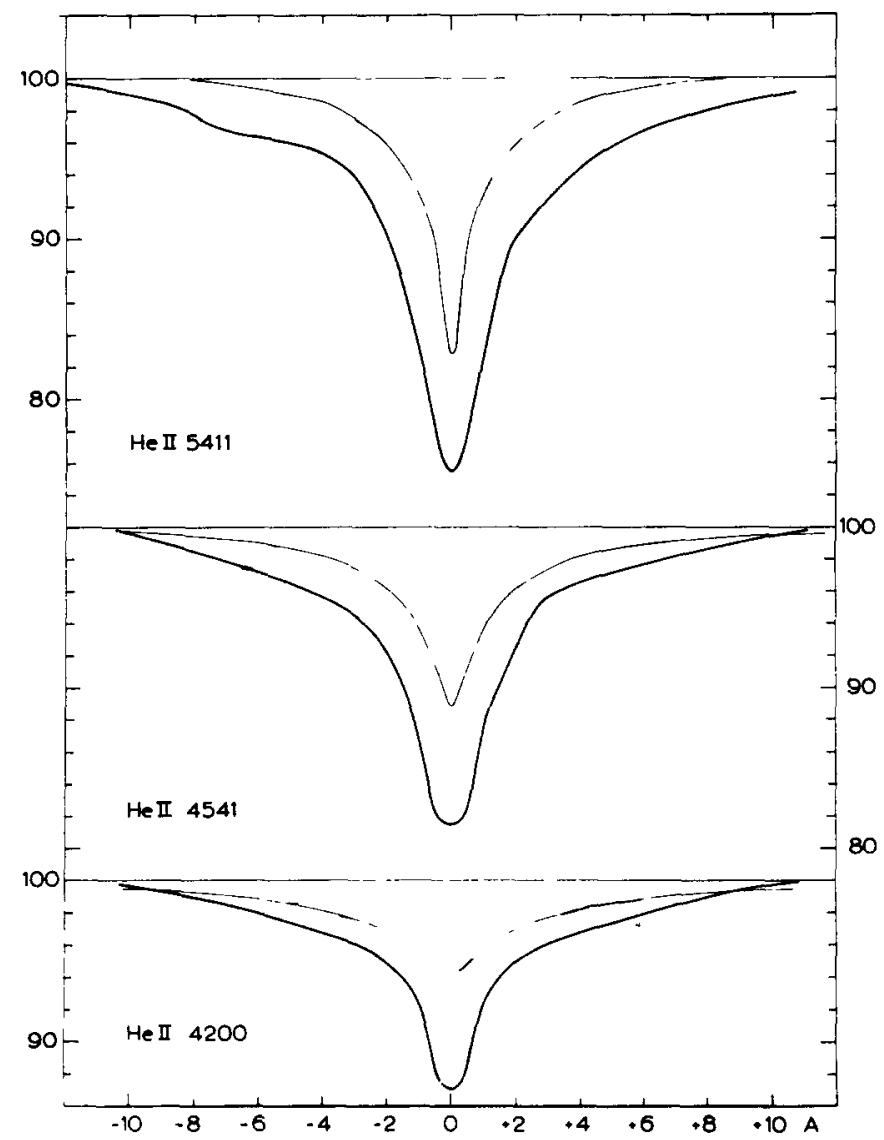

Fia. 4. The profiles of He II 5411, 4541, and 4200 in 10 Lacertae (thick lıne) compared with profiles predıct. ed using model PPB40 (thin line).

The predicted He II lines of the $n=4$ series are compared with the observed lines in Fig. 4. The observed lines have deeper and wider cores than the predicted lines. Introducing microturbulence into the line formation process would make the computed cores wider and slightly shallower, a change which would not remove the discrepancy at the centres of the lines. To match these lines one might consider using a model of earlier spectral type

Predicted and observed lines of $\mathrm{O} \Pi, \mathrm{O}$ III and Ne II are compared with observed profiles in 10 Lacertae in Fig. 5, while lines of $\mathrm{Mg} \mathrm{II}, \mathrm{Si}$ III and $\mathrm{Si} \mathrm{IV}$ are compared in Fig. 6. The predictions from model 98 are very nearly the same as those from model P7. The predictions, made using model PPB40 are, in principle, the best. In the case of $\mathrm{O} \mathrm{II}, \mathrm{O} \mathrm{m}, \mathrm{Ne} \mathrm{II}, \mathrm{Si}$ III and $\mathrm{Si} \mathrm{IV}$, the line blanketed model gives profiles which agree better with the observations than do the profiles predicted from unblanketed models. A velocity field of the order of 


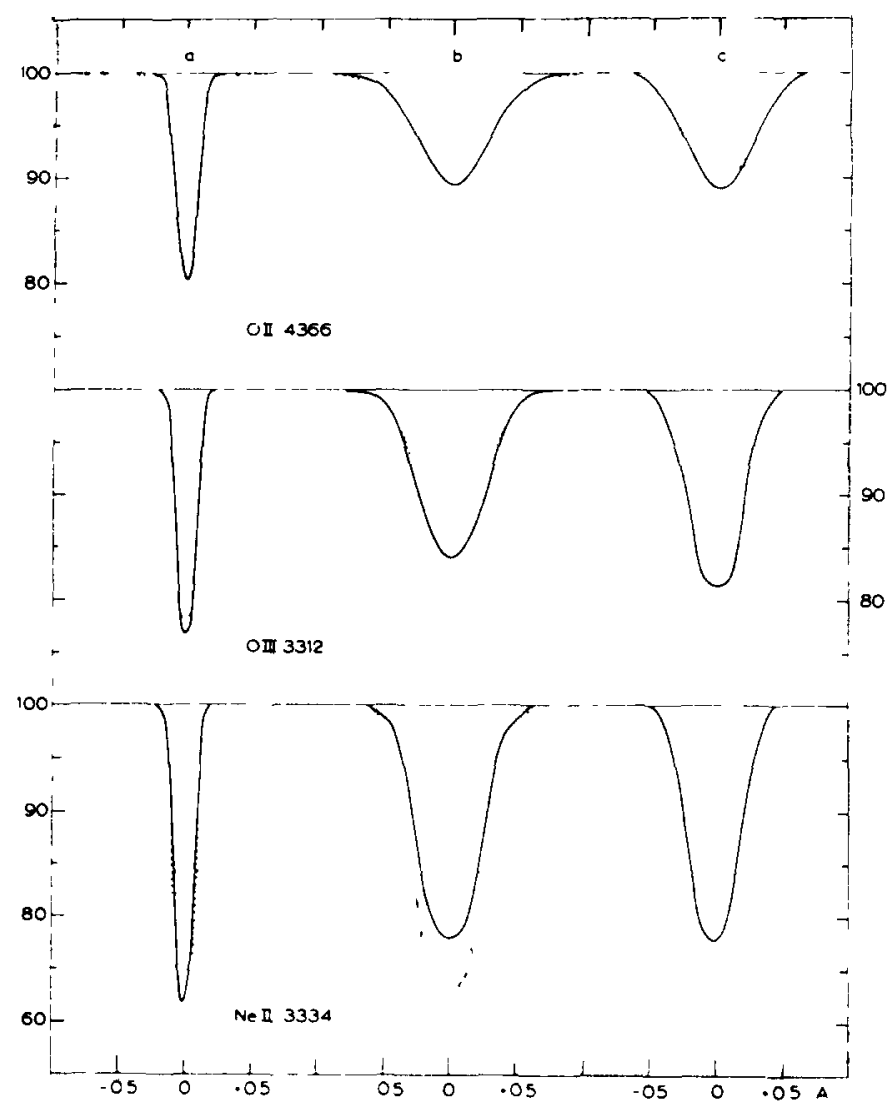

Fra. 5. Computed profiles of $\mathrm{O}$ Ir, $\mathrm{O}$ IIr and $\mathrm{Ne}$ II lines compared with the profiles observed in 10 Lacertae. Results from model PPB40 are shown by a solid line, those from model 98 by a broken line. Column $a$ gives the profiles with thermal Doppler broadening, column $b$ the profiles with 4 times $\Delta v_{D}$ and column $c$ the observed profiles.

4 times the thermal motion gives line widths and line depths closely resembling the observed values. The profiles predicted with only thermal motion and $10 \times$ the classical radiation damping do not resemble the observed profiles at all. In the case of the $\mathrm{OII}, \mathrm{O}$ III, and $\mathrm{Ne}$ II lines the adopted parameters result in lines which match the observed lines well. The observed $\mathrm{Mg}$ II blend at $\mathbf{4 4 8 1} \AA$ is very much stronger and deeper than can be predicted by any of the $\mathrm{O}$ type models. Sumilarly the Si III and Si Iv lines are observed to be deeper than the computed profiles obtamed with model PPB40. On the other hand, as reference to Table 3 will show, the computed profiles of the selected Fe mI lines (and, by inference, of Si II) are much shallower and weaker than the observed Si II and Fe IrI lines in the spectrum of 10 Lacertae even when microturbulence is introduced. Microturbulence has little effect on the computed equivalent widths of the Fe mI lines since these lines are so weak that they lie on the Doppler part of the curve of growth.

The conclusion of this eomparison is that the absorption line spectrum predicted using model PPB40 does not match well the spectrum of 10 Lacertae, although this predicted spectrum does match better when microturbulence is introduced than does that of model 98 


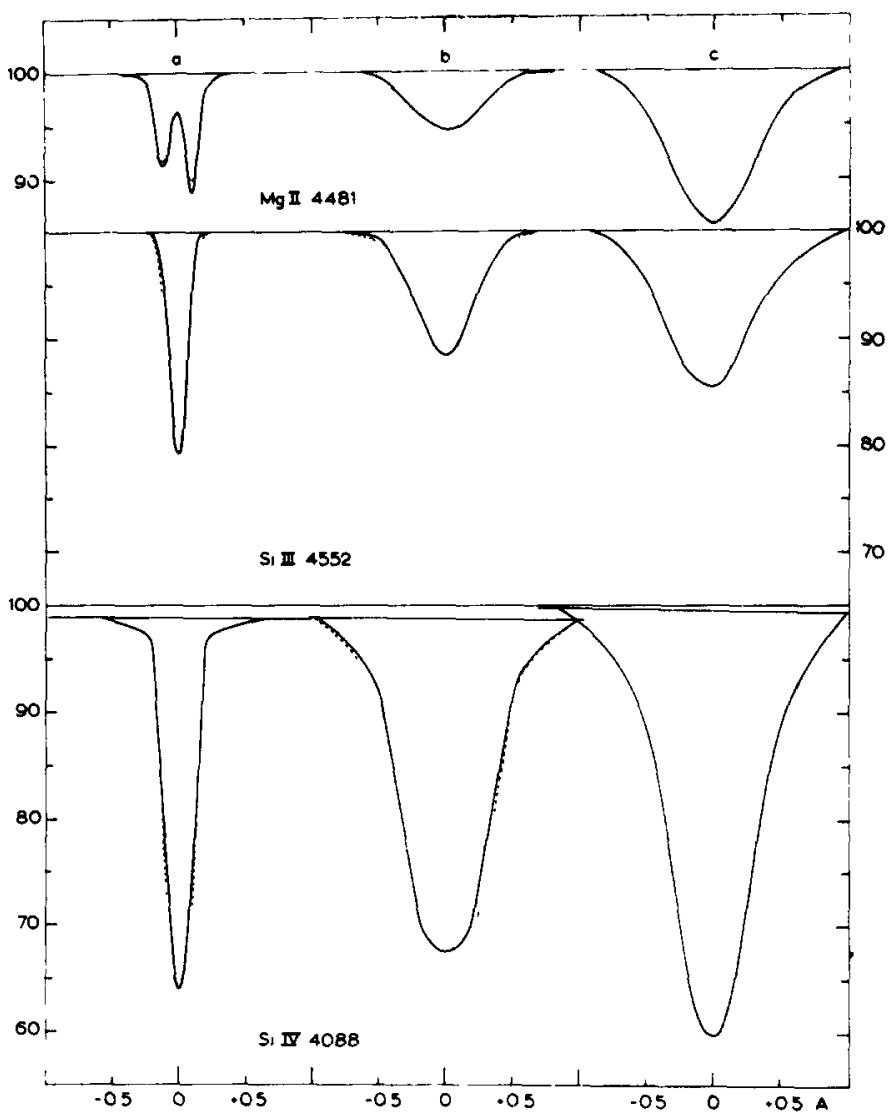

Fic. 6. Computed profiles of $\mathrm{Mg} \mathrm{II}, \mathrm{Si}$ III and Si IV lines compared with the profiles observed in 10 Lacertae. Results from model PPB40 are shown by a solid lune, those from model P7 by a broken ine. Column $a$ gives the profiles with thermal Doppler broadening, column $b$ the profiles with 4 times $v_{D}$ and column $c$ the observed profiles.

or of P7. The fact that the observed $\mathrm{H}$, Si $\Pi$, and $\mathrm{Fe} \mathrm{m}$ lines are too strong suggests that we are observing a source with an atmosphere at lower temperatures than given by the model, while the fact that the high excitation lines due to He II and Si IV are observed to be too strong argues for a hotter source. Possibly the spectrum of 10 Lacertae is composite. Irregular variations in the radial velocity are known and short-lived changes in the weak spectral lines occur sporadically (Grygar, 1964; van Helden, 1966). In Table 5 central depths are given for the $H \alpha, H \beta, H \gamma$, and $H \delta$ profiles computed using an unblanketed model (P8) which corresponds approximately to type B0 V. These central intensities correspond better to the observed central intensities in 10 Lacertae than do those computed using model PPB40.

A further point of concern is whether the adopted simple respresentation of the process of line formation is adequate, in particular for the strong absorption lines which are used to define the spectral type. Two basic assumptions have been made: (i) that the distribution of the atoms and ions over their possible energy states may be found using the equilibrium relationships known as Saha's and Boltzmann's laws, and (ii) that the re-emission in line- 
frequencies is given by Kirchhoff's relation. These relationshıps give explicit formulas which can be evaluated for each value of temperature and electron pressure. They are known to be valid in a gas which is sufficiently dense that the distribution over the possible energy states is dommated by collisional processes. Laboratory experience with plasmas at the temperatures considered here indicates that the necessary electron density for the above relationships to be valid are of the order of $10^{15}$ or greater (see, e.g., McWhirter, 1965) However, in the layers of the models from which the central parts of the $\mathrm{H}, \mathrm{He}, \mathrm{He} \Pi$, Si III, Si IV, and other strong lines come the electron density is less than $10^{14}$ (Underhill, 1968 a) and it may go as low as $10^{12}$. Clearly non-LTE populations of the various energy states of the atoms and ions are to be expected That non-LTE populations do occur un B-type model atmospheres has been demonstrated by Strom and Kalkofen (1966) and by Mihalas (1967) in connection with evaluating the continuous absorption coefficient of hydrogen and by Muhalas and Stone (1968) in the case of the contmuous absorption coefficient due to helium. Evidence is accumulating that the adopted simple theory of line formation is too simple for representing accurately the formation of the centers of strong lines.

\section{Theoretical Predictions vs. Observation for B Stars}

Five B-type model atmospheres were published by Underhill (1962a). Since then a few other unblanketed B-type models in the same series have been published (Underhill, 1963, 1968 a) while others remain unpublished. Information about the effective temperatures, bolometric corrections and approximate spectral types of some of the models have been published by Underhill $(1966 \mathrm{a}, \mathrm{b})$ In what follows computed equivalent widths and central intensities will be presented for a number of lines in model 119, model 66 and its improved equivalent P13, model 55 and its equivalent P12, model 63 and its equivalent P11, model 64 and its equivalent P8. Models 66,55,63, and 64 have been published in Underhill (1962a) as models 2, 3, 4, and 5 while model Pl3 is published in Underhill (1968b). The other models used here (119, P12, P11, and P8) are given in Tables A1, A2, A3, and A4. A line blanketed model which is closely equvalent in spectral type to model 63 has been published by Guillaume (1966); it is named B13. Miss Guillaume has published the profiles and equivalent widths of many lines in model B13; profiles and equivalent widths of some lines in models 66, 55, 63, and 64 are given by Underhill $(1962 \mathrm{~b})$ for some abundances, while other data about the lines of $\mathrm{Mg}$ II, Si II, Si III, and Si IV are given by Underhill (1963) and by De Groot and Underhill (1964) Detailed information about the intensity distribution in the continuous spectrum of models of types $\mathrm{B1.5}$ to $09 \mathrm{~V}$ is given by Underhill and Walker (1966)

Information about the equivalent widths and the central intensities of the hydrogen lines in B type model atmospheres is given in Tables 4 and 5 Here the results from the pico

Table 4 The Predicted Equivalent Widths of

Hydrogen Lines in B Type Model Atmospheres

\begin{tabular}{|c|c|c|c|c|c|}
\hline $\begin{array}{c}\text { Model } \\
T_{\text {eff }} \\
\log g\end{array}$ & $\begin{array}{c}119 \\
14,870^{\circ} \mathrm{K} \\
3 \cdot 7\end{array}$ & $\begin{array}{c}\mathrm{P} 13 \\
15,326^{\circ} \mathrm{K} \\
4 \cdot 0\end{array}$ & $\begin{array}{c}\mathrm{P} 12 \\
19,186^{\circ} \mathrm{K} \\
40\end{array}$ & $\begin{array}{c}\text { P11 } \\
25,653^{\circ} \mathrm{K} \\
4 \cdot 0\end{array}$ & $\begin{array}{c}\mathrm{P8} \\
31,053{ }^{\circ} \mathrm{K} \\
4 \cdot 0\end{array}$ \\
\hline $\mathrm{H} \alpha$ & - & $10 \cdot 84 \AA$ & $7 \cdot 311 \AA$ & $5.512 \AA$ & $3.877 \AA$ \\
\hline $\mathbf{H} \beta$ & $9 \cdot 109 \AA$ & $10 \cdot 31 \AA$ & $8 \cdot 170 \AA$ & $5 \cdot 327 \AA$ & $3 \cdot 443 \AA$ \\
\hline $\mathbf{H} \gamma$ & $9 \cdot 864 \AA$ & $11 \cdot 10 \AA$ & $8 \cdot 361 \AA$ & $5551 \AA$ & $3 \cdot 614^{a} \AA$ \\
\hline $\mathbf{H} \delta$ & $10 \cdot 46 \AA$ & $12.26 \AA$ & $8.830 \AA$ & $5.925 \AA$ & $3 \cdot 819^{\mathrm{a}} \AA$ \\
\hline
\end{tabular}

a Computed for model 64 which has $T_{\text {eff }}=31,023^{\circ}$ 
Table 5. The Predicted Central Intensitins of Hydrogen Lines in B Txpe Model Atmospheres

\begin{tabular}{c|c|c|c|c|c}
\hline Model & 119 & P13 & P12 & P11 & P8 \\
\hline H $\alpha$ & - & 0389 & 0.472 & 0.537 & 0.583 \\
H $\beta$ & $0 \cdot 258$ & 0.252 & $0 \cdot 340$ & 0.429 & 0.503 \\
H $\gamma$ & $0 \cdot 207$ & 0.202 & 0.290 & 0.386 & $0.587^{\mathrm{a}}$ \\
$\mathbf{H} \delta$ & 0.184 & 0181 & $\mathbf{0 . 2 6 9}$ & 0374 & $\mathbf{0 5 7 0 ^ { \mathrm { a } }}$ \\
\hline
\end{tabular}

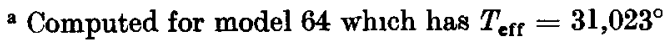

Table 6. Predicted Equivalent Widths (mÅ) of Lines in the Spectra of B Type MODEL ATMOSPHERES

\begin{tabular}{|c|c|c|c|c|c|c|c|c|c|c|c|c|}
\hline \multirow{2}{*}{$\begin{array}{r}\text { Mode } \\
T_{\text {ett }} \\
\log g \\
\text { line }\end{array}$} & & \multirow[b]{2}{*}{ of } & \multicolumn{2}{|c|}{$\begin{array}{c}119 \\
14,870^{\circ} \mathrm{K} \\
3 \cdot 7\end{array}$} & \multicolumn{2}{|c|}{$\begin{array}{c}66 \\
15,333^{\circ} \mathrm{K} \\
4 \cdot 0\end{array}$} & \multicolumn{2}{|c|}{$\begin{array}{c}55 \\
19,215^{\circ} \mathrm{K} \\
4 \cdot 0\end{array}$} & \multicolumn{2}{|c|}{$\begin{array}{c}63 \\
25,673^{\circ} \mathrm{K} \\
4 \cdot 0\end{array}$} & \multicolumn{2}{|c|}{$\begin{array}{c}64 \\
31,023^{\circ} \mathrm{K} \\
4 \cdot 0\end{array}$} \\
\hline & & & $1 \times \Delta v_{D}$ & $4 \times \Delta \nu_{D}$ & $1 \times \Delta v_{D}$ & $4 \times \Delta v_{D}$ & $1 \times \Delta v_{D}$ & $4 \times \Delta v_{D}$ & $1 \times \Delta y_{D}$ & $4 \times \Delta v_{D}$ & $1 \times \Delta v_{D}$ & $4 \times \Delta v_{D}$ \\
\hline \multirow[t]{5}{*}{$\mathrm{C}$ II } & 6582 & 0.622 & 42 & 83 & 40 & 76 & 100 & 251 & 155 & 460 & 107 & 269 \\
\hline & 65 & 124 & 55 & 121 & 51 & 110 & 119 & 320 & 170 & 523 & 118 & 350 \\
\hline & 4267 & a & 104 & 184 & 100 & 179 & 197 & 392 & 275 & 588 & 208 & 419 \\
\hline & 3920 & 0.572 & 50 & 114 & 50 & 112 & 93 & 250 & 127 & 360 & 84 & 192 \\
\hline & 3918 & 0.286 & 41 & 81 & 38 & 79 & 78 & 197 & 111 & 287 & 66 & 126 \\
\hline $\mathrm{Mg}_{\text {II }}$ & 4481 & a & & & 174 & & 136 & & 90 & & 47 & 65 \\
\hline \multirow[t]{2}{*}{ Si II } & 6371 & 0.947 & & & 120 & 336 & 96 & 295 & 67 & 162 & & \\
\hline & 6347 & 1.901 & & & 141 & 389 & 113 & 340 & 78 & 222 & & \\
\hline$+\mathrm{H} \delta$ & 4130 & $5 \cdot 94$ & 140 & & 131 & 310 & 129 & 297 & 89 & 227 & 9 & \\
\hline$+\mathbf{H} \delta$ & 4128 & $3 \cdot 96$ & 130 & & 132 & 294 & 112 & 263 & 80 & 209 & 8 & \\
\hline$+\mathbf{H}$ & 3862 & 0.120 & & & 117 & 315 & 97 & 249 & 34 & 81 & & \\
\hline$+\mathbf{H}$ & 3856 & 0.217 & & & 131 & 358 & 102 & 289 & 58 & 118 & & \\
\hline$+\mathrm{H}$ & 3853 & 0.024 & & & 92 & 198 & 56 & 121 & 18 & 21 & & \\
\hline \multirow[t]{3}{*}{ S1 III } & 4552 & 2075 & & & 20 & & 57 & & 111 & & 106 & 267 \\
\hline & 4568 & 1.243 & & & 17 & & 45 & & 112 & & 88 & \\
\hline & 4574 & $0 \cdot 414$ & & & 6 & & 31 & & 80 & & 71 & \\
\hline$S_{t} \mathrm{IV}$ & 4116 & 0.772 & & & & & 07 & & 23 & & 88 & 226 \\
\hline & 4088 & 1.544 & & & & & 0.7 & & 30 & & 93 & 262 \\
\hline
\end{tabular}

a For $4481 \cdot 13 g f$ is $5 \cdot 70$, for $4481 \cdot 33 g f$ is 380 ; for 42672 gf is $5 \cdot 608$; while for 42760 gf is 376 .

models (P) are used wherever possible, because these models give a better representation of the outermost layers of a star than do the original set of models. The predicted equivalent widths of lines of $\mathrm{C}$ II, Mg II, Si II, Si III, and Si IV in B-type model atmospheres are given in Table 6 for thermal Doppler broadening and for four times this. In all cases the damping constant was put equal to $10 \times$ the classical radiation damping constant. The central intensities for these cases are listed in Table 7. In the case of thermal Doppler broadening only the $\mathrm{Mg}$ II blend at $4481 \AA$ and the $\mathrm{C}$ II blend at $4267 \AA$ are partially resolved. The central intensities are as follows:

\begin{tabular}{c|c|c|c|c|c}
\hline Model & 119 & 66 & 55 & 63 & 64 \\
\hline $\mathbf{4 2 6 7 \cdot 0}$ & 0.731 & 0.742 & 0.570 & 0.505 & $0 \cdot 613$ \\
$\mathbf{4 2 6 7 \cdot 2}$ & 0.705 & 0.705 & 0.540 & $0 \cdot 499$ & $0 \cdot 605$ \\
$\mathbf{4 4 8 1 \cdot 1}$ & - & $\mathbf{0 . 3 4 3}$ & 0.469 & $0 \cdot 635$ & $0 \cdot 809$ \\
$\mathbf{4 4 8 1 3}$ & - & $\mathbf{0 . 3 5 2}$ & $\mathbf{0 . 4 8 4}$ & $\mathbf{0 . 6 6 3}$ & 0.835 \\
\hline
\end{tabular}

7 B-VTA Vol 13 
Table 7. Computed Central Intensities of Lines in the Spectra of B-Type Model ATMOSPHERES

\begin{tabular}{|c|c|c|c|c|c|c|c|c|c|c|c|}
\hline \multirow{2}{*}{\multicolumn{2}{|c|}{$\frac{\text { Model }}{\text { line }}$}} & \multicolumn{2}{|c|}{119} & \multicolumn{2}{|c|}{66} & \multicolumn{2}{|c|}{55} & \multicolumn{2}{|c|}{63} & \multicolumn{2}{|c|}{64} \\
\hline & & $1 \times \Delta \nu_{D}$ & $4 \times \Delta \nu_{D}$ & $1 \times \Delta v_{D}$ & $4 \times \Delta v_{D}$ & $1 \times \Delta v_{D}$ & $4 \times \Delta v_{D}$ & $1 \times \Delta v_{D}$ & $4 \times \Delta v_{D}$ & $1 \times \Delta v_{D}$ & $4 \times \Delta v_{D}$ \\
\hline \multirow[t]{5}{*}{ C II } & 6582 & 0833 & 0903 & 0840 & 0910 & 0684 & 0764 & 0665 & 0683 & 0740 & 0790 \\
\hline & 6578 & 0785 & 0872 & 0.795 & 0.878 & 0653 & 0.723 & 0661 & 0670 & 0.727 & 0758 \\
\hline & 4267 & a & 0.786 & $\mathbf{a}$ & 0786 & $\mathbf{a}$ & 0615 & a & 0.517 & a & 0635 \\
\hline & 3920 & 0699 & 0810 & 0.707 & 0815 & 0550 & 0655 & 0479 & 0545 & $0 \cdot 624$ & 0732 \\
\hline & 3918 & 0758 & 0.858 & 0.761 & 0861 & 0598 & 0705 & 0504 & 0605 & 0.665 & 0819 \\
\hline Mg II & 4481 & & & a & & $a$ & & a & & $\mathbf{a}$ & \\
\hline \multirow[t]{2}{*}{ S1 II } & 6371 & & & 0.511 & 0539 & 0595 & $0 \cdot 617$ & $0 \cdot 680$ & 0705 & & \\
\hline & 6347 & & & 0.504 & 0.519 & 0589 & 0601 & 0663 & $0 \cdot 753$ & & \\
\hline$+\mathbf{H} \delta$ & 4130 & 0250 & & $0 \cdot 292$ & 0.338 & $0 \cdot 383$ & $0 \cdot 415$ & 0.503 & 0580 & 0.943 & \\
\hline$+\mathbf{H} \delta$ & 4128 & 0236 & & 0286 & 0320 & $0 \cdot 380$ & 0402 & $0 \cdot 493$ & 0547 & 0924 & \\
\hline$+\mathrm{H}$ & 3862 & & & 0.257 & 0307 & 0359 & 0420 & 0569 & 0763 & & \\
\hline$+\mathbf{H}$ & 3856 & & & 0247 & 0278 & 0348 & $0 \cdot 384$ & 0511 & 0677 & & \\
\hline$+\mathrm{H}$ & 3853 & & & 0320 & $0 \cdot 449$ & $0 \cdot 437$ & 0626 & 0799 & 0935 & & \\
\hline Si III & 4552 & & & 0.858 & & 0670 & & 0.534 & & 0611 & 0631 \\
\hline & 4568 & & & 0885 & & 0704 & & 0.582 & & 0.617 & \\
\hline & 4574 & & & 0.932 & & 0.776 & & 0595 & & 0639 & \\
\hline Si IV & 4116 & & & & & 0.921 & & $0 \cdot 809$ & & 0602 & 0657 \\
\hline$+\mathbf{H} \delta$ & 4088 & & & & & 0905 & & 0.722 & & 0.584 & 0626 \\
\hline
\end{tabular}

See text.

Table 8. Equivalent Widths (mÅ) and Central Intensitins of 0 in AND III LINES IN B-Type Model ATMOSPHERES

\begin{tabular}{|c|c|c|c|c|c|c|c|}
\hline \multicolumn{2}{|c|}{ Model } & 55 & 63 & 63 & 55 & 63 & 63 \\
\hline \multicolumn{2}{|c|}{ line } & $4 \times \Delta v_{D}$ & $1 \times \Delta y_{D}$ & $4 \times \Delta y_{D}$ & $4 \times \Delta v_{D}$ & $1 \times \Delta v_{D}$ & $4 \times \Delta v_{D}$ \\
\hline \multirow[t]{2}{*}{ O II } & 4676 & 33 & 78 & 173 & 0.946 & 0.674 & $0 \cdot 762$ \\
\hline & 4661 & 35 & 64 & 200 & 0.940 & 0.663 & 0.748 \\
\hline$+\mathrm{H} \gamma$ & 4366 & 37 & 65 & 191 & 0.920 & 0640 & $0 \cdot 729$ \\
\hline$+\mathrm{H}_{\gamma}$ & 4349 & 40 & 70 & 202 & 0816 & 0590 & 0669 \\
\hline \multirow[t]{6}{*}{ Fe III } & 40531 & 0.9 & $4 \cdot 9$ & $5 \cdot 4$ & 0.996 & 0926 & 0977 \\
\hline & $4137 \cdot 8$ & $2 \cdot 7$ & 120 & 185 & 0.989 & 0.849 & 0.932 \\
\hline & $4139 \cdot 4$ & $2 \cdot 0$ & 105 & 148 & 0992 & 0.865 & $0 \cdot 944$ \\
\hline & 41405 & 09 & $5 \cdot 1$ & 69 & 0997 & 0.925 & 0.976 \\
\hline & $4164 \cdot 7$ & 46 & 165 & $35 \cdot 0$ & 0982 & 0802 & $0 \cdot 897$ \\
\hline & 42386 & 05 & 31 & 41 & 0998 & 0954 & 0986 \\
\hline
\end{tabular}

Data about lines of $\mathrm{O}$ II and Fe III are given in Table 8 In some cases results for other abundances are given in the references listed above. It is easy to see from Tables 6 and 8 that the introduction of microturbulence of the order of $5-15 \mathrm{~km} / \mathrm{sec}$ will produce a significant change in the predicted equivalent widths The changes in line depth are less marked

It is difficult to assign an equivalent spectral type to a model because one does not have available the same information which is used to classify real stars. The following tabulation indicates the uncertanties which exist The spectral type given in column 4 is found from the computed size of the Balmer jump, $D$, (no lines) using an empirical relation which may be established between the value of $D$ measured by Chalonge and Divan and the MK spectral type (see Underhill, 1966a), while the spectral type given in column 5 is found by 
entering Fig. 1 with the effective temperature found from the integrated flux and reading off the equivalent absorption-line spectral type. A rough allowance for $U V$ line blanketing will reduce the listed effective temperature by 10 per cent and make the spectral type somewhat later.

\begin{tabular}{|c|c|c|c|c|c|}
\hline \multirow{2}{*}{$\begin{array}{c}\text { Model } \\
\text { No. }\end{array}$} & \multirow{2}{*}{$\begin{array}{l}T_{\text {eft }} \\
\left({ }^{\circ} \mathbf{K}\right)\end{array}$} & \multirow{2}{*}{$\log g$} & \multicolumn{2}{|c|}{ Spectral type } & \multirow{2}{*}{$H \gamma$ fits } \\
\hline & & & from $D$ & from $T_{\text {eft }}$ & \\
\hline 119 & 14,870 & $3 \cdot 7$ & $B 7$ & B5 & $\tau$ Herculis B5 ${ }^{\text {IV }}$ \\
\hline (P13) 66 & 15,333 & $4 \cdot 0$ & B6 & B4.5 & (5 Draconis B6 III) \\
\hline (P12) 55 & 19,215 & 40 & B3 & B2 & ¿ Herculis B3 ${ }^{\mathrm{v}}$ \\
\hline (P11) 63 & 25,673 & 4.0 & B2 & B0.5 & $\gamma$ Pegasi B2 VI \\
\hline (P8) & 31,023 & 4.0 & B0 & B0 & \\
\hline
\end{tabular}

Profiles of $H \gamma$ in the spectrum of $\gamma$ Pegasi B2 IV and of $\iota$ Herculis B3 V have been published by Wright, Lee, Jacobsen, and Greenstein (1963) while Heintze (1968) has published a profile of $H \gamma$ in the spectrum of $\tau$ Herculis B5 IV. The $H \gamma$ profile of $\gamma$ Pegasi fits that predicted from model P11 well to $\Delta \lambda= \pm 7 \AA$. At larger distances from the centre of the line the computed wings are stronger than the observed wings Probably a model with smaller $\log g$ would be appropriate, but the observed wings appear "clipped" as if the continuum had been drawn too low by about 2 per cent. The observed $H \gamma$ profile in $\iota$ Herculis fits that predicted using model P12 fairly well, but again one suspects that a smaller value of $g$ would give a better fit and that the observed continuum should be raised slightly. Heintze (1968) has shown that model 119 gives a rather good representation of $H \gamma \mathrm{m} \tau$ Herculis. Thus the models presented here can be identified with known sharp-lined stars as indicated in the above tabulation. The identıfication of $\zeta$ Draconis with model 66 (P13) is tentative, being based on preliminary material. These identifications confirm that the shape of the $H \gamma$ profile and the Balmer jump tend to indicate a consistent spectral type for a model. However, taking the effective temperature of a model, and entering a diagram which relates effective temperature to line spectral type does not result in the same spectral type for the model, particularly if the model is unblanketed as in the present cases. The line-blanketed model of Miss Guillaume (1966) gives an $H \gamma$ profile which is nearly identical with that from model P11. Furthermore P11 and B13 have about the same Balmer jump. Thus either model, according to these two criteria, will represent $\gamma$ Pegası equally well. Model B13 has $\log g$ $=4.0$ and its effective temperature is $23,255^{\circ} \mathrm{K}$

Observed equivalent widths in sharp-lined main-sequence $B$ stars are given in Table 9 for comparison with the computed values given in Tables 6 and 8. The values for $\zeta$ Draconis are preliminary. They are an unweighted mean of results published by Underhill (1948) and of results derived at Utrecht from two high-dispersion spectrograms obtained by Underhll with the coudé spectrograph of the 48-inch telescope at the Dominion Astrophysical Observatory and from two spectrograms obtained by Heintze with the coudé spectrograph of the 200-inch telescope at Mount Palomar. The material for $\iota$ Herculis is an unweighted mean of the values given by Wright, Lee, Jacobsen, and Greenstein (1963) and those from a list due to Aller (1966). The results for 3 Centauri A are from the list by Jugaku, Sargent, and Greenstem (1961). This material is included because it is interesting to see how the spectrum of this peculiar star compares with that of normal B stars and with the predicted line strengths. The results for $\gamma$ Pegasi are an unweighted mean of the results published by Underhill (1948), Aller (1949), Aller and Jugaku (1958), Wright, Lee, Jacobsen, and Greenstein (1963) and in addition for Fe II of values derived at Utrecht from spectrograms 
Table 9. Obsmenved Equivalent Widths (m§) of Lines in B Type Spectra

\begin{tabular}{|c|c|c|c|c|c|c|}
\hline & ne & \ Draconis & $\iota$ Herculis & 3 Centaurı A & $\gamma$ Pegasi & $\tau$ Scorpu \\
\hline $\mathrm{C}_{\text {II }}$ & 6582 & 89 & 137 & 46 & 130 & 61 \\
\hline & 6578 & 90 & 127 & 44 & 167 & 35 \\
\hline & $4267^{a}$ & 167 & 168 & 110 & 209 & 82 \\
\hline & 3920 & 62 & 80 & 41 & 91 & 39 \\
\hline & 3918 & 52 & 70 & 38 & 78 & 40 \\
\hline O II & 4676 & - & - & - & 51 & 89 \\
\hline & 4661 & - & 13 & - & 54 & 96 \\
\hline & 4366 & - & 16 & - & 51 & 70 \\
\hline & 4349 & - & 14 & - & 61 & 99 \\
\hline $\mathrm{Mg} \mathrm{II}$ & $4481^{a}$ & 418 & 200 & 141 & 160 & 90 \\
\hline S1 II & 6371 & 246 & - & 100 & 38 & - \\
\hline & 6347 & 344 & - & 116 & 65 & - \\
\hline & 4130 & 192 & 63 & 74 & 34 & - \\
\hline & 4128 & 176 & 12 & 64 & 38 & - \\
\hline & 3862 & 156 & 55 & 88 & 42 & - \\
\hline & 3856 & 154 & 70 & 96 & 32 & - \\
\hline & 3853 & 128 & 31 & 55 & 14 & - \\
\hline S1 III & 4552 & 146 & 46 & 53 & 134 & 132 \\
\hline & 4568 & 52 & 34 & 42 & 104 & 109 \\
\hline & 4574 & 64 & 23 & 24 & 75 & 70 \\
\hline S1 IV & 4116 & - & - & - & 15 & 127 \\
\hline & 4088 & - & 一 & 一 & 17 & 137 \\
\hline Fe III & 4238.6 & - & - & - & 62 & - \\
\hline & 4164.7 & - & 11 & - & 46 & 14 \\
\hline & 41405 & - & 4 & - & 18 & 118 \\
\hline & 41393 & - & 5 & - & 25 & - \\
\hline & 4137.8 & - & 10 & 一 & 30 & - \\
\hline & $4053 \cdot 1$ & $\ldots$ & - & - & 24 & - \\
\hline
\end{tabular}

a Equivalent width for both lines is listed.

obtained by Underhill at the Dominion Astrophysical Observatory. The results for $\tau$ Scorpi are from the list by Scholz (1965). It is impossible at this time to determine what systematic errors, if any, exist between the various published equivalent widths. The true value of each listed equivalent width probably les within $\pm 20 \mathrm{~m} \AA$ of the given number, but in some cases, notably Fe III, the results may be seriously disturbed by blends which have not been resolved.

The observed equvalent widths will now be compared with the computed values given in Tables 6 and 8 The detailed model atmosphere study of 3 Centauri A by Hardorp (1966) indicates that model 55 is the model of the present group which should fit 3 Centauri A best. Hardorp's preferred model has an effective temperature of $19,386^{\circ} \mathrm{K}$ and $\log g=3 \cdot 87$. The $H \gamma$ profile for $\tau$ Scorpii published by Scholz (1967) fits that predicted from model P8 better in the core than the profile predicted by Scholz from a model with slightly higher effective temperature, thus it is not inappropriate to compare $\tau$ Scorpii with model 64 (P8). It would be better if theoretical results were also available for a set of models with $\log g=3 \cdot 7$. In the case of $\mathrm{C} \amalg$ the computed equivalent widths from model 119 and from model 66 are nearly the same. On the basis of this evidence we shall assume that the predicted equivalent widths for the rather weak lines would not be strongly affected by changes in $\log g$ of the order of $\pm 0 \cdot 3$. 
$\zeta$ Draconis. The equivalent widths of the $\mathrm{C} \Pi$ lines, $\mathrm{Mg} \amalg 4481$, and $\mathrm{Si} \Pi \mathbf{I} 6371,6347$ can be matched using model 66 (or 119), the adopted abundances and $g f$ values, and a velocity field between 3 and 4 times the thermal velocity distribution However $\mathrm{Si}$ II 4128, 4130, and $3862,3856,3853$ are then predicted to be stronger than observed. The Si III lines are predicted to be significantly weaker than observed. In the case of $\mathrm{Si}$ m introducing microturbulence would produce only a small strengthening of the line, since these lines, according to the theory which has been used, are close to the Doppler part of the curve of growth The observed strengths of the Si III lines in $\zeta$ Draconis are clearly inconsistent with the theory; this problem deserves further study. Some remarks about the shape of the lines in $\zeta$ Draconis are given below

¿ Herculis. The comparison is made with model 55. The results obtained using thermal Doppler broadening match better than those with 4 times this A definite conclusion about the presence of microturbulence, and how much, can best be made after profiles are compared. The $\mathrm{C}$ II lines are observed to be somewhat weaker than the predicted lines for the case of no microturbulence except in the case of the red lines. The observed 0 II lines are definitely weaker than the predicted values with microturbulence, but they might match well with predictions for only thermal broadening were these available. The $\mathrm{Mg}$ II doublet at $4481 \AA$ is significantly stronger than the predicted doublet with thermal broadening only. The match with Si II 4128,4130 and with $3862,3856,3853$ is poor, the predicted lines bemg too strong. The predicted Si III lines, also for the case of thermal broadening only, are stronger than what is observed. One solution for the discrepancies with the Si II and Si III lines might be to reduce the adopted silicon abundance. However, the effects of changing $\log g$ should also be investigated. The observed strengths of the Fe min lines are not consistent with the predictions from model 55. An increase in the abundance of iron by about a factor 4 seems to be indicated

3 Centauri $A$. The comparison is made with model 55 for the case of no microturbulence since model 55 has a temperature structure closely resembling that of the model considered to be best by Hardorp (1966) The $\mathrm{C} \Pi$ lines are observed to be definitely weaker than what has been predicted, suggesting a smaller abundance of carbon. The $\mathrm{Mg}$ II line matches well as do all the Si II lines except 4128 and 4130 which are predicted to be too strong. The strengths of the Si $\mathrm{mI}$ lines are also well reproduced by model 55 and the adopted abundance of silicon. A decision about the amount of microturbulence can best be made from a study of the profiles of the lines. Hardorp (1966) has suggested a microturbulence of $4 \mathrm{~km} / \mathrm{sec}$, which is about 1.33 times the most probable thermal velocity for $\mathrm{Si}$ at a temperature of $15,000^{\circ} \mathrm{K}$. To take account of a microturbulence of this order, we should compare with results computed using model 55 and Doppler broadening corresponding to $2 \cdot 3 \Delta v_{D}$ The discrepancy between the predicted and observed $\mathrm{C}$ m equivalent widths would then become greater and one would wish to reduce the abundances of magnesium and silicon. The discrepancy with Si II 4128 and 4130 would remain.

$\gamma$ Pegasi The comparison is made with model 63. The case of thermal broadening gives the best overall fit, but a definitive conclusion about the presence of microturbulence in $\gamma$ Pegasi can only be made after a detailed study has been made of the line profiles. It is known that the lines of $\gamma$ Pegasi are not so sharp as theory predicts in the case of thermal Doppler broadening only. The observed C II lines are somewhat weaker, on the average, than the computed lines, which suggests that perhaps the abundance of carbon has been overestimated by a small amount. The observed $\mathrm{O}$ II lines are significantly weaker than predicted. The observed strength of $\mathrm{Mg} \Pi 4481$ is significantly greater than the predicted strength. The match with the Si II lines is fairly good except for 4128 and 4130 which are 
observed to be significantly weaker than predicted. The match in equivalent width for the Si III and Si IV lines is fairly good. The Fe III lines are observed to be significantly stronger than predicted suggesting that an increase in the adopted abundance of $\mathrm{Fe}$ is needed. A study at Utrecht by M. A J. Snijders indicates that an increase in $\mathrm{Ngf}$ by a factor 4 may not be unreasonable

$\tau$ Scorpii The comparison is made with model 64. Since the lines of $\tau$ Scorpi are known to be very sharp it is appropriate to compare with the predictions made for the case of thermal Doppler broadening only, i e. microturbulence zero. The observed $\mathrm{C}$ II lines are significantly weaker than the predicted lines, suggesting either that the abundance of carbon should be reduced by a significant factor or that a hotter model be used. However, if a hotter model is used, then the hydrogen spectrum will fit very poorly. The $O$ m lines were not predicted using model 64 . The observed equivalent widths are stronger than the values predicted using model 63 or model PPB40 (Table 3). Probably the $\mathrm{O} \Pi$ lines reach maximum strength in a model having characteristics intermediate between those of model 63 and model 64 The $\mathrm{Mg} \amalg \mathbf{4 4 8 1}$ blend is observed to be considerably stronger than the line predicted with model 64. The Si II lines were not observed by Scholz. The Si III and S1 IV lines are stronger than predicted with model 64 for the case of no microturbulence A better fit in equivalent width might be obtained by introducing some microturbulence. Scholz (1967) has suggested a microturbulence of $5 \mathrm{~km} / \mathrm{sec}$, which seems reasonable.

These comparisons between observed equivalent widths in sharp-lined main-sequence B stars and the predictions from models lead to the following conclusions:

(i) The C II lines are generally predicted to be too strong, except in the case of the comparison between $\zeta$ Draconis and model 66. The adopted Coulomb approximation $g f$ values for the $\mathrm{C}$ II lines agree reasonably well with the experimental results of Roberts and Eckerle (1967).

(ii) The Mg II line is predicted to be too weak in $\iota$ Herculis, $\gamma$ Pegasi, and $\tau$ Scorpii, but the predictions fit well for 3 Centauri A and probably would fit well for $\zeta$ Draconis if a velocity field about 35 times the thermal Doppler motion was introduced.

(ui) The match with the Si II lines is fairly good except that 4128 and 4130 are consistently predicted to be stronger than observed (cf Underhill, 1968b). Whether this result is due to an error in the adopted $g f$ values or to a breakdown of the adopted theory of line formation for this multiplet cannot be decided at present.

(iv) The Si Irr lines are predicted to be too weak in the case of $\zeta$ Draconis and $\tau$ Scorpii. They match well in the case of 3 Centauri A and $\gamma$ Pegasi, but are predicted to be too strong in the case of $\iota$ Herculis. It is suspected that the level populations of Si III depart from LTE values under some circumstances (Underhill, 1963), thus the adopted theory of line formation is suspect for the Si III lines.

(v) The predicted Si Iv lines match fairly well in the case of $\gamma$ Pegasi where they are weak, but for $\tau$ Scorpi, where they are strong, the predicted lines in the case of no microturbulence are too weak This behaviour is similar to that found for 10 Lacertae. Introducing microturbulence leads to a better fit between observed and predicted profiles

(vi) The predicted lines of Fe III are too weak. An mcrease in the parameter $\mathrm{Ngf}$ by a factor of the order of 4 is required to obtain a fair agreement between the observed and predicted line strengths. The adopted $g f$ values were obtained by the Coulomb approximation 
Sharp conclusions about the abundances of the elements in $O$ and $B$ type stars cannot be drawn from comparisons such as these until it has been shown that the simple theory of line formation described in section $3 \mathrm{~A}$ is adequate for the selected lines. In the present cases the correct value of microturbulence to use should be settled by a study of line profiles, attention being paid to the effects of rotation of the star and to the choice of transfer equation to represent the process of line formation.

\section{Factors Affecting the Shapes of the Line Profiles}

The theoretical line profiles dıscussed so far have been obtained by assuming that the contribution to the emissivity resulting from absorption in line frequencies may be represented by $l_{v} \varrho B_{v}$, where $B_{v}$ is the Planck function, $\varrho$ is the density, and $l_{v}$ is the line absorption coefficient. Another extreme assumption is to consider that the emissivity results from coherent isotropic scattering. Then the contribution is $l_{v} \varrho J_{v}$. The difference in profile which results can be seen by comparing the narrowest profiles in columns (a) and (b) of Fig. 7. Here calculated profiles are given of $\mathrm{Si}$ III 4552 in model B13, which is due to

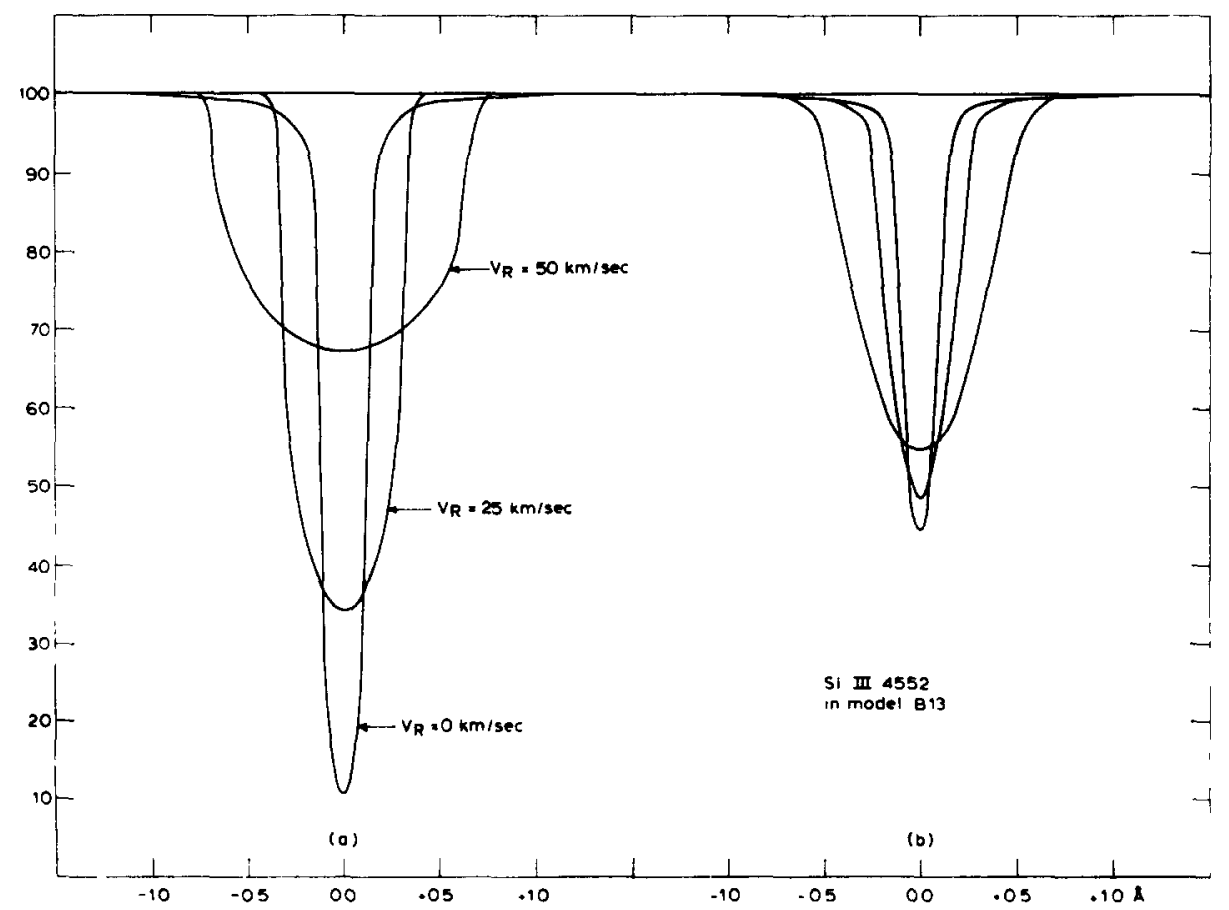

FTa. 7. Profiles of $S_{1}$ III 4552 in model B13: $a$ for the case that the emissivity is $l_{\nu} \varrho J_{v}$ and the projected rotational velocity is 0,25 , and $50 \mathrm{~km} / \mathrm{sec} ; b$ for the case that the emussivity is $l_{\nu} \varrho B_{v}$ and for thermal Doppler broadening, $2 \times$ and $4 \times$ this.

Miss Guillaume (1966). This model is a line-blanketed equivalent of model 63. The abundance of silicon is that of Table 2 and the adopted $g f$ value is given in Table 3.

Rotation of the star may cause a widening of the profile, and so may the presence of microturbulence. The rotationally broadened profiles of column (a) of Fig. 7 have been derived using the theory of Unsolld (1955) assuming that the unbroadened line has negligible width and that the limb-darkening coefficient is 0.5 . These profiles give a first ap- 
proximation to the changes in shape that would be caused by rotation of the star The equivalent width of the line is not changed from the value for the line computed by the isotropic, coherent scattering approximation, when the projected rotational velocity $v_{R}$ is zero.

The effects of introducing microturbulence are llustrated by the profiles of column (b) of Fig. 7. Here the contribution of the line to the emissivaty is $l_{v} \varrho B_{v}$ and the profiles have been calculated for the case of thermal Doppler broadenung, 2 times and 4 times this

The following equivalent widths and central intensities are found.

\begin{tabular}{l|c|c|c|c|c}
\hline \multirow{2}{*}{ Emissivity } & Model 63 & \multicolumn{3}{|c|}{ Model B13 } & Model B13 \\
\cline { 2 - 6 } & $l_{\nu} \varrho B_{\nu}$ & \multicolumn{3}{|c|}{$l_{\nu} \varrho B_{\nu}$} & $l_{\nu} \varrho J_{v}$ \\
\hline Doppler broadening & $1 \times$ & $1 \times$ & $2 \times$ & $4 \times$ & $1 \times$ \\
Equivalent width (mA) & 111 & 128 & 206 & 335 & 376 \\
Central intensity & 0.534 & 0445 & 0.486 & 0.547 & $0 \cdot 106$ \\
\hline
\end{tabular}

First it is clear that the line-blanketed model gives a slightly stronger and slightly deeper line than does the unblanketed model. Secondly, introducing microturbulence (increased Doppler broadening) makes this rather strong line even stronger, considerably broader, and shallower. These changes in profile are exactly what were predicted by Struve and Elvey (1934) from simple theoretical considerations. Changing to isotropic coherent scattering as the mode of line formation produces a much deeper and stronger line for the same number of atoms In fact in the scattering mode the equivalent widh for the case of no microturbulence is about 10 per cent greater than that for the case of microturbulence equivalent to 3 times the thermal Doppler motion If one was certain that the star did not rotate, then one could reject the scattering mode for line formation because no lines in normal B stars are observed to be so deep as the computations give. However, the rotationally broadened profiles of Fig 7 (a) (which have been obtaned by an approximate theory) lead to the inference that a rotation of about $35 \mathrm{~km} / \mathrm{sec}$ and the scattering mode of line formation would result in a line very similar in profile to that computed for the thermodynamic equilıbrium mode of line formation and a total Doppler broadening 4 times the thermal broadening Clearly it is going to be dufficult to separate the effects of the mode of line formation and of rotation or of microturbulence on the profiles and equivalent widths of single lines

The above example has been presented to demonstrate that the modest depths of absorption lines in B stars cannot a priori be taken as evidence that line formation according to a scattering mode is excluded One must be certain also about the rotation of the star before excluding scattering. Isotropic coherent scattering is an extreme simplification of the problem of line formation and it is not physically probable. However, if the population of the energy states into which the atoms and ions can be excited are not to be described by thermal equilibrium expressions such as Saha's and Boltzmann's laws, then one must turn to a scattering formulation for the line formation problem

In order to explore the changes in line shape resulting from the slow rotation of a star, the problem of rotational broadening has been examined in detail (Underhill, 1968c). Some results for the line Si $I 6347$ in model P13 are given in Figs. 8 and 9 where they are compared with the observed profile of Si II 6347 in $\zeta$ Draconis. The profiles in Fig. 8 illustrate the changes which occur when one starts with a line which is broadened by thermal Doppler broadening only. Figure 9 gives the results for a line which is broadening by 4 times the 


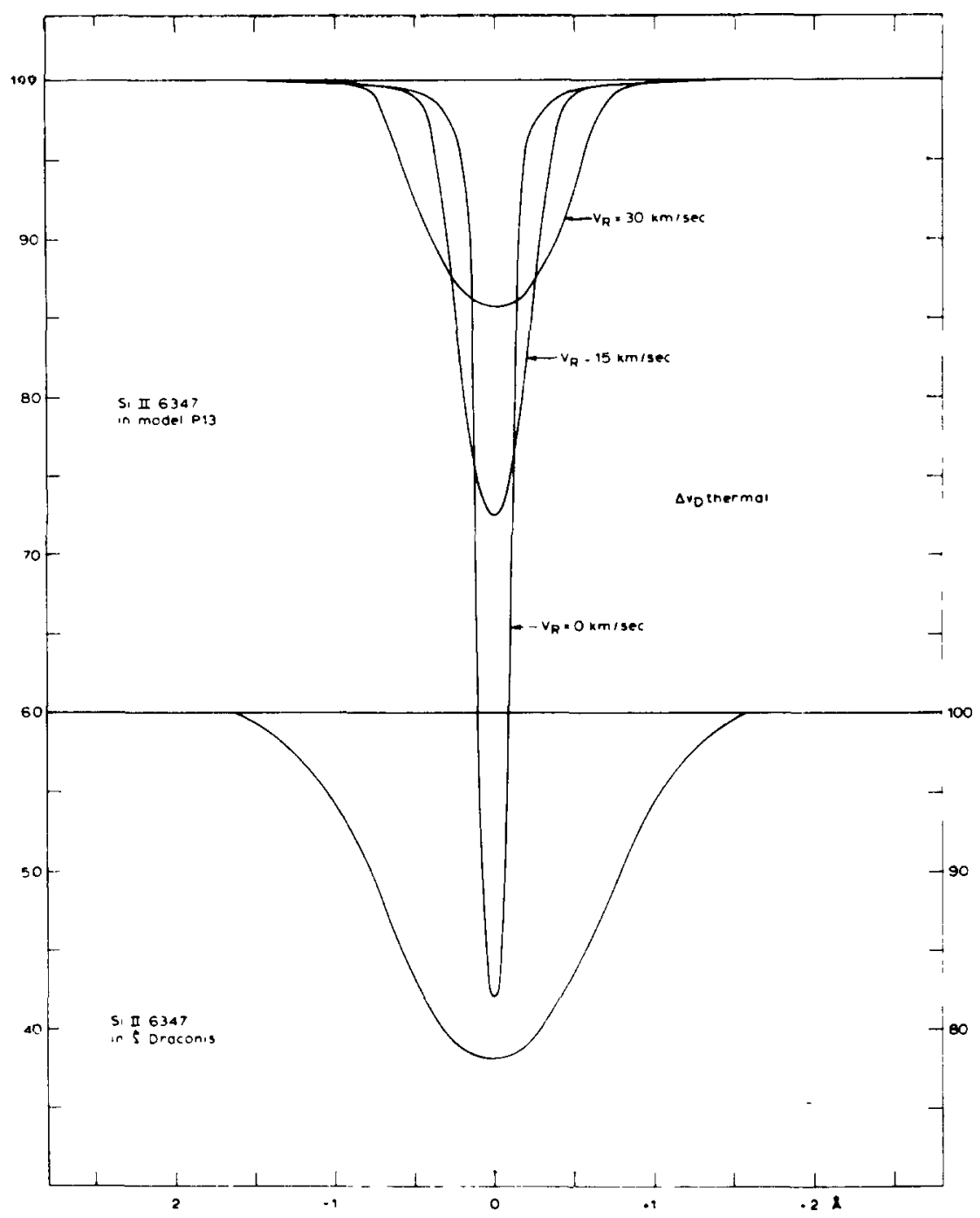

Fra. 8. Computed rotationally broadened profiles of $S_{1}$ II 6347 in model P13 for the case of no microturbulence and emissivity $l_{\nu} \varrho B_{v}$ compared with the observed profile for $\zeta$ Draconıs.

thermal Doppler broadening. The typical dish-shaped profile does not appear until the velocity displacement due to the rotation velocity $v_{R}$ is significantly larger than the half halfwidth of the undistorted line. The observed Si II line in $\zeta$ Draconis is best represented by the case $v_{R}=60 \mathrm{~km} / \mathrm{sec}$ and 4 times the thermal Doppler broadening. These profiles have all been calculated for the case that the emissivity in the line is given by $l_{\nu} \varrho B_{v}$. Another result would have been obtained had the calculations started from a scattering formulation for the line formation problem. There is very little difference found between the profile resulting from the above more exact formulation of the problem of broadening by rotation and the profile resulting from Unsöld's method when $v_{R} \lambda / c$ is greater than twice the half halfwidth of the unbroadened line. The detalled theory must be used for small values of the rotational velocity.

7a B-VIA Vol 13 


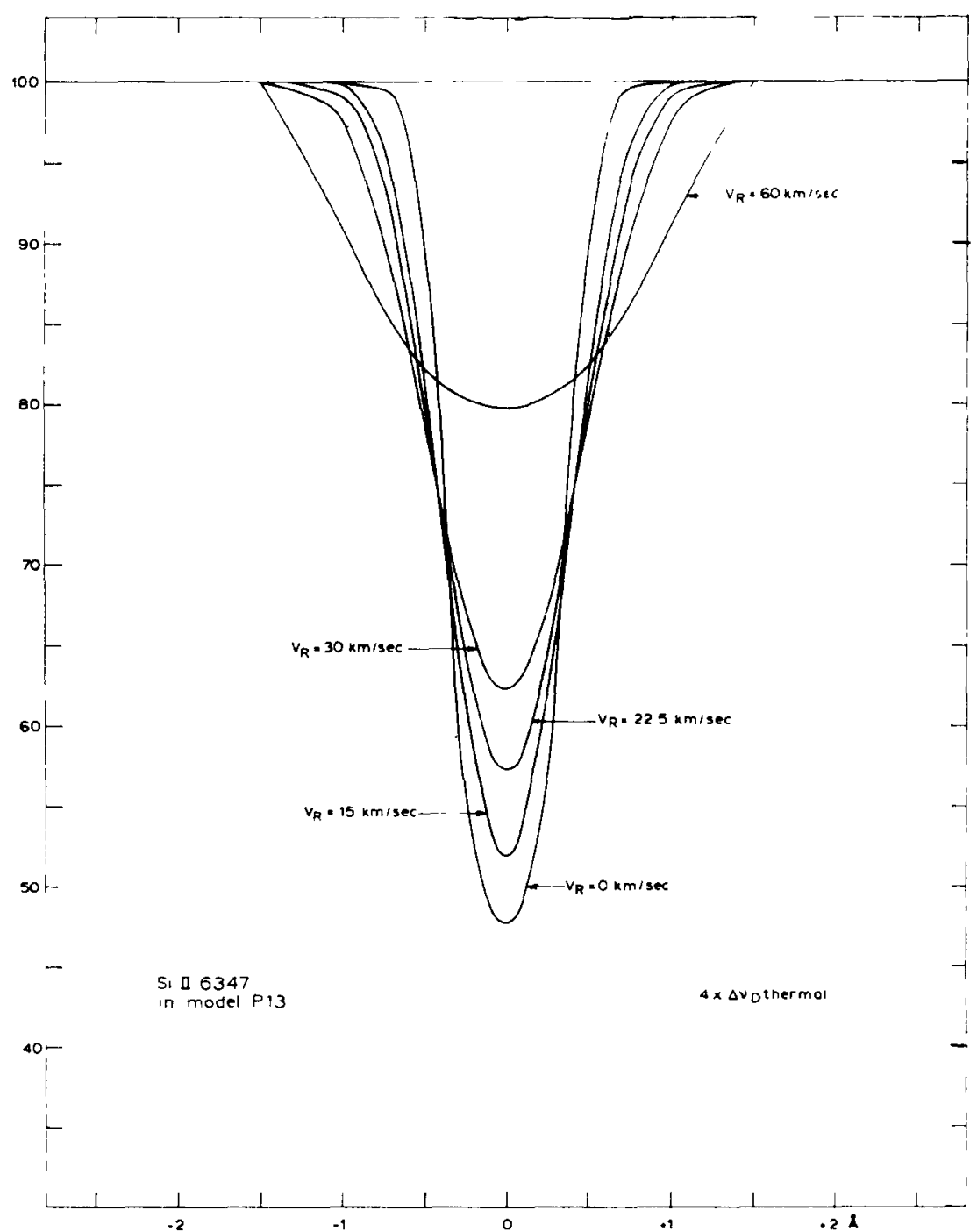

Fia. 9. Computed rotationally broadened profiles of $\mathrm{S}_{1}$ II 6347 in mode P13 for the case of $4 \times$ thermal Doppler broadening and the emissivity is $l_{v} \varrho B_{v}$.

\section{E. The Meaning of Spectral Type at Types $B$ and $O$}

In sections 3B and 3C details in observed stellar spectra have been compared with spectra predicted using model atmospheres and a rather simple theory of line formation based on the concept that the atoms, ions, and radiation in the stellar atmosphere interact as though thermodynamic equilibrium exists at each point in the atmosphere. In the case of the stars of types B0 and earlier, the relative slope of the continuous spectrum in the spectral region $3500-6700 \AA$ is found to be rather insensitive to the details of the model and to the effective temperature This means that photometric criteria for establishing spectral type cannot give sharp results for O-stars even if a correction for interstellar reddening can be applied with confidence 
The chief lines used for classifying the $\mathrm{O}$ stars are those of $\mathrm{H}, \mathrm{He}, \mathrm{He}$ II and $\mathrm{Si}$ Iv. The models and the adopted theory of line formation do not give a satisfactory and consistent representation of what is observed. In fact it appears that the apparent strengths of the prominent absorption lines in 0 type stars are the result of factors not yet included in the theory. In particular it seems to be very doubtful if the ratio of the strengths of the He I to the He $\mathrm{Ir}$ lines in absorption, which is the chef criterion for classifying $\mathrm{O}$ stars, is dominantly a function of effective temperature. Rather, the effects of departures from local thermodynamic equilibrium and, perhaps, of motion of the gases in the outer atmosphere must be taken into account before a consustent physical interpretation can be given of the meaning of the subdivisions of class 0 .

In the case of the B stars similar uncertainties exist in the interpretation of the empurically selected spectral type criteria. When the effective temperature is less than $20,000^{\circ} \mathrm{K}$, the shape of the continuous spectrum and the shape of the wings of $H \gamma$ can be used to obtain a consistent set of the parameters $T_{\text {eff }}$ and $\log g$ for a $B$ star. However, when the weaker lines in the spectrum are compared with those predicted from a model which represents the hydrogen spectrum rather well, various inconsistent results appear The stronger lines which are used as secondary criteria for spectral type are formed so high in the atmosphere that doubt exists concerning the validity of the simple theory which is used. This remark is particularly applicable to interpretations of the strengths of the $\mathrm{He} \mathrm{I}$ lines (which have not been investigated here, but which are known to be sensitive to departures of the level populations from thermal equilibrium values), and to other strong lines. In section $3 \mathrm{D}$ attention was drawn to the difficulty of deciding upon a unique way of interpreting the line strengths and profiles A set of spectral types based on the hydrogen spectrum only can be related in a unique way with the parameters $T_{\text {eff }}$ and $\log g$. However, spectral types based upon the relative strengths of the $\mathrm{He} I$ and other lines may easily be in confluct with the "hydrogen" types because the strengths of some of the other lines appear to be rather sensitive to the details of the process of line formation. This means that the problem of interpreting absorption line spectral types in terms of effective temperature, acceleration of gravity and abundances is by no means solved in a unique manner

\section{Evidence for Departures from Looal Thermodynamio Equitibrium in Early TYPe StaRs}

In a detailed and comprehensive paper Milne (1930) described the basic physical problems which are encountered when one attempts to predict stellar spectra. He concluded that we have four different weapons at our disposal:

I. The method of pure thermodynamics.

II. The method of statistical mechanics.

III. The method of detailed mechanisms.

IV. The principle of detailed balancing.

The theories that have been applied so far belong to methods I and II. However, the results yielded have not been entirely satisfactory and we are forced to consider using method III. To use method III satisfactorily we need a rather complete theory of the interaction of radiation with matter although we can use IV to reduce the work involved. That this type of attack might prove to be necessary was foreseen by Milne. In this section an attempt will be made to define more closely what detailed mechanisms should be considered when interpreting the spectra of early type stars Several formulas have been 
developed for the case of optically thin laboratory plasmas to estimate the electron density above which, for a given electron temperature, one may be sure that sufficient collisions occur that the excitation and ionization of the gas may be described by Saha's and Boltzmann's laws (see, for instance, McWhirter, 1965) A typical formula is of the form

$$
N_{e} \geqq C T_{e}^{\frac{1}{2}} \chi(p, q) \times 10^{12} \text { per } \mathrm{cm}^{3},
$$

where $C$ is a constant, typical values of which range from 0.92 to 1.6 depending on exactly what condition is taken to represent effective equilibrium level populations, and $\chi(p, q)$ is the excitation energy in volts between the levels $p$ and $q$ which are under consideration. Thus in a late $B$ type atmosphere where a typical electron temperature 1 s $10,000^{\circ}$ and for an excitation energy of $10 \mathrm{~V}$, the electron density must be greater than $10^{15}$ before one can expect the level populations to be given correctly by the Saha and Boltzmann laws. In $O$ stars where the temperature is higher and one is concerned with excitation energies of the order of $30 \mathrm{~V}$ or more, the required electron density is even greater. Stellar atmospheres may be optically thick in some lines; thus the above formula cannot be applied rigorously, but it does indicate that one should not be surprised if those parts of the stellar spectrum which are formed in regions where the electron density is less than $10^{15}$ indicate that non-equilibrium level populations occur. Under these circumstances the emissivity term in the equation of radiative transfer should be formulated carefully according to the appropriate laws of physics. This means that a term having the characteristics of a scattering emissivity should be introduced.

Table 10 The Electron Density, $N_{e}$, at Various Depths in the Models

\begin{tabular}{|c|c|c|c|c|c|c|}
\hline$\tau$ & $\begin{array}{c}\text { Model } \\
119\end{array}$ & $\begin{array}{c}\text { Model } \\
66\end{array}$ & $\begin{array}{l}\text { Model } \\
\mathbf{5 5}\end{array}$ & $\begin{array}{c}\text { Model } \\
\quad 63\end{array}$ & $\begin{array}{l}\text { Model } \\
\quad 64\end{array}$ & $\begin{array}{l}\text { Model } \\
\text { PPB40 }\end{array}$ \\
\hline 000 & $7 \cdot 40+12^{\mathrm{a}}$ & $1.50+13^{\mathrm{a}}$ & $2 \cdot 24+13^{\mathrm{a}}$ & $3 \cdot 00+13^{\mathrm{a}}$ & $3 \cdot 15+13^{a}$ & $137+11^{\mathrm{a}}$ \\
\hline 0.01 & $1.52+13$ & $2 \cdot 66+13$ & $3 \cdot 74+13$ & $5 \cdot 18+13$ & $5 \cdot 61+13$ & $2 \cdot 04+13$ \\
\hline 002 & $213+13$ & $3 \cdot 45+13$ & $5 \cdot 02+13$ & $708+13$ & $7 \cdot 75+13$ & $4 \cdot 03+13$ \\
\hline 0.05 & $3.46+13$ & $543+13$ & $8 \cdot 44+13$ & $1 \cdot 20+14$ & $1 \cdot 30+14$ & $8 \cdot 56+13$ \\
\hline $0 \cdot 10$ & $5 \cdot 37+13$ & $8 \cdot 55+13$ & $1 \cdot 32+14$ & $1.86+14$ & $2 \cdot 00+14$ & $147+14$ \\
\hline $0 \cdot 20$ & $8 \cdot 63+13$ & $1 \cdot 35+14$ & $2 \cdot 08+14$ & $2 \cdot 94+14$ & $3.03+14$ & $2 \cdot 42+14$ \\
\hline 030 & $1 \cdot 14+14$ & $1.82+14$ & $2 \cdot 74+14$ & $3 \cdot 87+14$ & $3 \cdot 89+14$ & $3 \cdot 15+14$ \\
\hline $0 \cdot 40$ & $1 \cdot 42+14$ & $2 \cdot 26+14$ & $3 \cdot 38+14$ & $4 \cdot 69+14$ & $462+14$ & $3 \cdot 78+14$ \\
\hline 0.50 & $1 \cdot 69+14$ & $2 \cdot 69+14$ & $4 \cdot 01+14$ & $5 \cdot 44+14$ & $5 \cdot 28+14$ & $434+14$ \\
\hline
\end{tabular}

a Power of 10 by which to multıply the first number.

Typical electron densities in B- and O-type model atmospheres are listed as a function of the characteristic optical depth in Table 10 The level $\tau=0.00$ is schematic; the precise values reached at the outer edge of the atmosphere depend upon how fine the steps in $\tau$ were as $\tau \rightarrow 0$. The listed values are upper limits. By examining the quadrature formulas used to evaluate the emergent flux $F_{v}(0)$ at any frequency in the spectrum one finds that most of the weight of the solutions falls near layers where $t_{v} \approx 0.4$ Thus if lone formation is assumed to occur as though local thermodynamic equilibrium exists, the values of $T$ and $N_{e}$ at the depth $t_{v} \approx 0.4$ are very significant in determining the value of $F_{v}(0)$.

Examination of the output from the Theoretical Spectrum Program which has been used to predict the results reported here shows that throughout class $B$, the continuous spectrum in the red $(\lambda \approx 6500 \AA)$ is determined by the $T$ and $N_{e}$ of layers near $\tau \approx 0 \cdot 2$, while the continuous spectrum near $4000 \AA$ is determined by the $T$ and $N_{e}$ of layers near 
$\tau=0.4$. The central parts of strong and moderately strong lines are determined by the $T$ and $N_{e}$ in the layers between $\tau=0.00$ and $\tau=0 \cdot 10$. Some examples referring to particular lines in particular models are given in Underhill $(1968,1969)$. From Table 10 one can see that $N_{e}$ is usually less than $5 \times 10^{14}$ in the layers where the observed parts of the spectra of $\mathrm{O}$ and B stars are formed, and that $N_{e}$ ss of the order of $10^{13}$ or lower in the regions where the cores of the strong absorption lines are formed. The conclusion to be drawn is obvious: the assumption that line formation can be handled by formulas appropriate for a state of local thermodynamic equilibrium cannot be justified. It is necessary to make use of Mulne's weapon III in order to obtain a sound understanding of the meaning of the absorption-line spectra of $O$ and $B$ stars. This problem, so far as it concerns predicting the continuous spectrum, has already been attacked by Kalkofen and Strom (1966) and by Mihalas (1967). These authors are currently continuing their study of these problems. In passing it should be noted that the strong lines found in the ultraviolet spectrum of $B$ stars (911-6-1500 $\AA$ ) are most certainly formed at levels near $\tau \approx 0.0$ Consequently a non-LTE theory of line formation should be used to predict these lines. Results such as those obtained by Morton (1965) using LTE theory can only be indicative of what may be expected. They certainly are not definitive.

The fact that many conspicuous details in 0 - and B-type spectra are the result of the action of particular processes has been known for a long time. These processes may be described as dilution effects, as optical pumping by radiation (fluorescent effects), as optical pumping by collision and as particular ionzation effects including autoionization.

Dilution effects. By this is meant that absorption lines coming from metastable levels, or from levels closely linked to metastable levels, are stronger in comparison to the lines from normal levels than is observed to be the case in the majority of stars of the same (rough) spectral type. The majority of stars are considered to have "normal" spectra and it is generally assumed that "normal" spectra correspond to the state of local thermodynamic equilibrium. Ionic and atomic spectra known to contain metastable levels and to show dilution effects are $\mathrm{He}$ I, $\mathrm{C}$ III, N IV, $\mathrm{O}_{\mathrm{I}}$, and $\mathrm{Si}$ m. Dilution effects are also said to occur in the second spectra of the metals. The most conspicuous cases of dilution effects are found in the extended atmospheres of supergiants, shell stars, Of and Wolf-Rayet stars. Less conspicuous cases occur in the spectra of main-sequence stars.

Optical pumping by radiation. By this is meant the selective filling of one particular energy level as the result of absorption of radiation of one particular wavelength. Wellknown cases in stellar atmospheres are the Bowen mechanisms for exciting the emission of $\mathrm{N}$ III 4634, 40,4l and He II 4686 in planetary nebulae and in the atmospheres of $\mathrm{O}$ and Wolf-Rayet stars. In cool stars various "mutilated multiplets" of Fe $I$ and the resonance lines of In I appear in emission due to coincidences between particular emission line intensities and the separation of the energy levels in the radiating atom.

Optical pumping by collision. In this case an atom or ion in the ground state collides with an abundant species in an excited state and the excitation energy is transferred during the collision. The result is that the atoms or the ions of the first species are selectively excited to one particular energy level from which they decay with the emission of line radiation. This process is well known for exciting some laser transitions and for exciting many forbidden lines in planetary nebulae. This process may be invoked for causing the particularly strong N IV 4057 emission in some Of and Wolf-Rayet stars (Underhill, 1967).

Particular ionization effects. These are similar in principle to optical pumping by radiation and by collision and they may perturb the degree of ionization of certain species from the values predicted by Saha's law. The process proposed by Underhill (1957) for exciting C m 
in emission in cool Of stars as a result of ionization by $\mathrm{He}$ II 303 is one example of what may occur. The fact that unusually strong lines of $\mathbf{P}$ II in late B-type spectra are correlated with unusually weak lines of $\mathrm{He}$ I can be explained as a result of ionization caused by collusion with neutral helium atoms in the metastable $2^{3} \mathrm{~S}$ level (Underhill, 1968d). Many atoms are known to possess doubly-excited states which autolonize. When the density in an atmosphere is sufficiently low that the degree of ionization is not determined solely by collisional processes, then the amount of ionzation that can occur as a consequence of a transition from the ground level, or other low-lying level of the atom to a doubly-excited state which has a large possıbllity for autoionization, this transition occurring as a result of absorption of radiation in that part of the stellar radiation field which is intense, can be important. It should be taken into account when determining the degree of Ionization of any species of atom in a stellar atmosphere One wonders whether it is merely a random coincidence that many of the elements deduced to be abnormally abundant in Am and Ap atmospheres using LTE theories of line formation are known to possess strong autoionizing levels in the atom. The abundances are usually deduced from the intensities of lines in the spectrum of the first ion. If the lonizing process sketched here is particularly effective and the number of recombinations is not in thermal balance, one can see that under certain atmospheric conditions the atoms might be preferentially driven to their first ionized state. If this process does occur at a significant rate, then the results of a standard analysis of the strengths of the lines of the first or of the second spectrum using LTE theories has little real meaning since the degree of ionization is not given by Saha's law.

The fact that processes of the above types are known to occur at significant rates in stars with somewhat extended atmospheres and the fact that the model atmospheres for mainsequence stars show that the electron density in the line-forming regions is low enough that one may doubt that thermodynamic equllbrium is fully established everywhere, point very clearly to the need for using Milne's weapon III, the method of detailed mechanisms, in order to predict the strength of the absorption lines in the spectra of early-type stars The inconsistencies between spectral types assigned on the basis of photometric measurements and those assigned according to the empirically selected relative intensities of rather strong lines are evidence of the same thing-early-type spectra cannot be explained in a consistent manner using only the concept of local thermodynamic equilıbrium.

\section{The Present Postion Regarding Interpreting Early-type Spectra}

\section{A. Main-sequence Stars}

The classical work with model atmospheres (see the review by Pecker (1965) for references) is based on the idea that local thermodynamic equllbrium is a concept which is satisfactory for simplifying the problem of predicting the spectrum which will emerge from a star. It has proved possible to use the shape of the continuous spectrum and the shape of the wings of $H \gamma$ (which is an indicator of the electron pressure in the layers where the continuous spectrum is formed) to find pairs of the parameters $T_{\text {eff }}$ and $\log g$ which will define a model representing rather well the continuous spectrum of a main-sequence star of type B2 or later. However, because of weaknesses in the theory of spectrum formation it is not usually possible to find a unique pair $\left(T_{\text {eff }}, \log g\right.$ ) in this way (Heintze, 1968, 1969). When more details of the spectrum are examined and compared with predicted spectra, it is usually impossible to obtain a good match in all details. The analysis of sections 3 and 4 has indicated that a more elaborate and physically correct theory of line formation should be used if one wishes 
to obtain meaningful conclusions about the abundances of the elements and the physical conditions in $\mathrm{O}$ - and B-type atmospheres. The indications are that these considerations are important to at least type A5.

The present comparisons between computed and observed spectra suggest that many of the particular differences between the absorption-line spectrum of a "peculiar" star and a "normal" star of about the same photometric or hydrogen spectral type may be due largely to different departures from the state of local thermodynamic equilibrium in the two cases. Slow rotation may be an important factor affecting the appearance of the line profiles, and tending to obscure recognition of the dominant physical mode of line formation

With the stars of spectral types B1 to 05 the interpretation problem is even more difficult. The shape of the continuous spectrum in the accessible spectral range is not a sensitive index of the effective temperature and all prominent spectral lines are formed under conditions such that detailed processes of excitation and ionization play a rôle. Consequently spectra of types $\mathrm{Bl}$ to $\mathrm{O5}$ cannot be interpreted accurately by means of theories based on thermodynamic concepts alone. Improved theories are essential if even rather rough interpretations are to be given in a consistent manner for the stars of types $\mathrm{Bl}$ to $\mathrm{O5}$

\section{B. Supergiants}

The interpretation of the spectra of early-type supergiants has barely begun. It is known that the absorption lines are usually broad and often have the rather square, steep-sided shape associated with microturbulence in the atmosphere. The strongest lines, by their displacements, indicate the presence of large stream motions in the atmosphere. Qualitatively one can argue that because of the lower electron density in the atmosphere, shown by the extended Balmer series, the departures from local thermodynamic equilibrium will be large in supergiant atmospheres Theories of line formation which take account of particular processes of ionization and excitation will be needed before much progress can be made with a quantitative analysis of supergiant spectra. It is an open question whether main-sequence and supergiant stars having the same spectral type, say B3, have closely the same effective temperature. The shape of the continuous spectrum is not a very useful tool for estimating $T_{\text {eff }}$ because large corrections for interstellar reddening have to be introduced in most cases, and furthermore it seems probable that the non-LTE effects discussed by Kalkofen and Strom and by Mihalas may be significant.

\section{Shell Stars, Of Stars, and Wolf-Rayet Stars}

The spectroscopic details which lead to certain stars being placed in these classes are dominantly the result of monochromatic radiative processes and of particular collisional processes. These stars have extended atmospheres which are opaque only in line frequencies. The amount of material in the line of sight does not seem to be enough to obscure the underlying main-sequence stellar spectrum at wavelengths in the contmuous spectrum between the Balmer and the Paschen jumps. Consequently any precise, quantitative interpretation of the spectrum of these stars must be based upon theories which take into account particular processes of line formation. Such theories are not yet available in general.

The emission lines originate from general recombination cascades and from particular processes of excitation. When an attempt is made to interpret the spectra of shell stars, Of stars, and Wolf-Rayet stars, attention must be paid to the fact that the observable geometrical extent of the star may differ by a significant amount in neighbouring radia- 
tions, and to the effects of motion in the outer atmosphere. Because of these complications the quantitative study of these spectra has not progressed far. However, the comparison of these spectra with the spectra of normal main-sequence stars provides an empirical indication of which spectral lines are sensitive to non-LTE conditions. A survey of how the line strengths and displacements vary with time, in the case of a few stars which vary, gives further empurical information about how the densities and radiation fields which exist in extended atmospheres react upon the atoms and ions there In those cases where enough shell absorption lines are observed that one can make use of the simple theory of a curve of growth for an absorption tube or for a Schuster atmosphere, prelıminary estimates can be made of the density and temperature in the shell. Such numbers are little more than parameters, but they do set limits withun which spectroscopic phenomena can be observed. Estimates of abundances can be very misleading because the classical method of estimating abundances by means of the curve of growth is based on the use of Saha's and Boltzmann's laws to relate the number of atoms in an excited level to the total abundance of the element, and these laws are not valid in extended atmospheres.

\section{Acknowledgements}

The results of this project to determme model atmospheres and to predict theoretical spectra for $\mathrm{O}$ and $\mathrm{B}$ stars have been obtained with the continued interest and support of several Institutes. Some of the model atmospheres and some of the theoretical spectra were obtained at the Computing Centre of the University of Washıngton, Seattle, with financial support from the Department of Mines and Technical Surveys, Canada, while I was still on the staff of the Dominion Astrophysical Observatory, Victoria, B C The major part of the computing work was done at the Institute of Space Studies, NASA, New York, to whom I am sincerely grateful for long continued support. The investigation of the ultraviolet spectra of some of the models and the development of a method for constructing lineblanketed models was supported by ESRO and carried out with the assistance of Dr K. G Lenhart at the ESDAC Computing centre The study of rotationally broadened profiles was done partly at Utrecht with the assistance of the University Computing Centre, and partly at the Joint Institute for Laboratory Astrophysics, University of Colorado. To the staff of these Institutes and Computing Centres I express my sincere gratitude for generous help

The observed line profiles have been obtamed from high-dispersion spectrograms obtained at the Dominion Astrophysical Observatory, at the Mount Wilson and Palomar Observatories and at the Kitt Peak Observatory.

The observational part of the general plan to compare predicted and observed line profiles in order to advance our understanding of early-type spectra is not yet complete. Most of the spectrograms have been studied at Utrecht and I am particularly grateful to M. de Groot, $R$ van Helden and $M A$. J. Snijders who have helped with various parts of the study A final word of thanks must go to Mrs G den Boggende-Dorresteljn who has assisted since 1962 with the plotting and planumetering of innumerable calculated line profiles and with the work with the Utrecht microphotometers, to Mrs J. Odijk-Nijenhuis who has typed all the tables and manuscript with great accuracy, and to Mr. R. Staleman for the drawings. 
TABLe A1. Modir 119

\begin{tabular}{|c|c|c|c|c|c|c|c|}
\hline$\tau$ & $\begin{array}{c}T \\
\text { (degrees) }\end{array}$ & $\begin{array}{r}P_{g} \\
\text { (dynes/c }\end{array}$ & $\left.m^{2}\right)$ & $\underset{\text { (dynes/cr }}{P_{e}}$ & $\left.m^{2}\right)$ & $\begin{array}{c}\overline{x+\sigma} \\
\left(\mathrm{cm}^{2} / \mathrm{g}\right)\end{array}$ & $\begin{array}{c}\sigma \\
\left(\mathrm{cm}^{2} / \mathrm{g}\right)\end{array}$ \\
\hline 0.00 & 8579 & 2222 & $1^{\mathrm{a}}$ & $8 \cdot 762$ & $0^{\mathrm{a}}$ & $2 \cdot 074$ & $0 \cdot 1965$ \\
\hline 0.01 & 9871 & 4503 & 1 & $2 \cdot 066$ & 1 & $1 \cdot 797$ & 02557 \\
\hline 0.02 & 10158 & $6 \cdot 484$ & 1 & $2 \cdot 986$ & 1 & $2 \cdot 286$ & 02576 \\
\hline 0.03 & 10376 & 8110 & 1 & $3 \cdot 748$ & 1 & $2 \cdot 721$ & 0.2593 \\
\hline 004 & 10571 & $9 \cdot 589$ & 1 & $4 \cdot 446$ & 1 & 2.952 & $0 \cdot 2608$ \\
\hline 0.05 & 10758 & $1 \cdot 104$ & 2 & $5 \cdot 134$ & 1 & 2.993 & 0.2621 \\
\hline 0.06 & 10917 & $1 \cdot 247$ & 2 & 5.806 & 1 & 3.063 & $0 \cdot 2630$ \\
\hline 0.07 & 11049 & $1 \cdot 387$ & 2 & $6 \cdot 467$ & 1 & $3 \cdot 115$ & 0.2637 \\
\hline 008 & 11189 & 1.527 & 2 & $7 \cdot 129$ & 1 & $3 \cdot 110$ & $0 \cdot 2644$ \\
\hline 0.09 & 11300 & $1 \cdot 666$ & 2 & $7 \cdot 787$ & 1 & $3 \cdot 134$ & $0 \cdot 2648$ \\
\hline 010 & 11393 & $1 \cdot 804$ & 2 & 8.347 & 1 & $3 \cdot 167$ & 0.2651 \\
\hline 020 & 12166 & $3 \cdot 085$ & 2 & $1 \cdot 449$ & 2 & $3 \cdot 385$ & 0.2672 \\
\hline 0.30 & 12730 & 4267 & 2 & $2 \cdot 009$ & 2 & 3.624 & $0 \cdot 2685$ \\
\hline 0.40 & 13270 & $5 \cdot 489$ & 2 & $2 \cdot 592$ & 2 & 3.515 & 02699 \\
\hline 050 & 13684 & 6.737 & 2 & $3 \cdot 189$ & 2 & 3448 & 02711 \\
\hline 060 & 14089 & 8.010 & 2 & 3.803 & 2 & $3 \cdot 374$ & $0 \cdot 2727$ \\
\hline 0.70 & 14462 & $9 \cdot 299$ & 2 & $4 \cdot 431$ & 2 & $3 \cdot 318$ & 0.2746 \\
\hline 0.80 & 14820 & 1.057 & 3 & 5058 & 2 & $3 \cdot 332$ & 0.2768 \\
\hline 0.90 & 15146 & $1 \cdot 185$ & 3 & $5 \cdot 695$ & 2 & $3 \cdot 303$ & $0 \cdot 2791$ \\
\hline 100 & 15480 & $1 \cdot 316$ & 3 & $6 \cdot 352$ & 2 & $3 \cdot 225$ & 0.2817 \\
\hline 1.5 & 16865 & 1982 & 3 & $9 \cdot 743$ & 2 & 3188 & 0.2918 \\
\hline $2 \cdot 0$ & 17910 & $2 \cdot 692$ & 3 & $1 \cdot 334$ & 3 & 3049 & 02964 \\
\hline $2 \cdot 5$ & 18887 & 3411 & 3 & 1698 & 3 & 3029 & 02989 \\
\hline $3 \cdot 0$ & 19627 & $4 \cdot 150$ & 3 & 2069 & 3 & 2.979 & 0.2999 \\
\hline 3.5 & 20274 & $4 \cdot 886$ & 3 & $2 \cdot 438$ & 3 & $2 \cdot 997$ & $0 \cdot 3004$ \\
\hline 40 & 20841 & $5 \cdot 612$ & 3 & 2.802 & 3 & 3037 & 03007 \\
\hline $5 \cdot 0$ & 21869 & 7.081 & 3 & $3 \cdot 537$ & 3 & 3.010 & $0 \cdot 3011$ \\
\hline $6 \cdot 0$ & 22781 & $8 \cdot 518$ & 3 & $4 \cdot 256$ & 3 & 3070 & 0.3013 \\
\hline $7 \cdot 0$ & 23623 & $9 \cdot 962$ & 3 & 4.979 & 3 & $3 \cdot 059$ & $0 \cdot 3014$ \\
\hline $8 \cdot 0$ & 24342 & $1 \cdot 138$ & 4 & $5 \cdot 689$ & 3 & 3104 & $0 \cdot 3015$ \\
\hline 90 & 25013 & 1.278 & 4 & $6 \cdot 390$ & 3 & 3142 & 0.3016 \\
\hline 10.0 & 25607 & 1.417 & 4 & 7083 & 3 & $3 \cdot 176$ & 0.3016 \\
\hline $15 \cdot 0$ & 28318 & 2.082 & 4 & 1041 & 4 & $3 \cdot 290$ & 0.3017 \\
\hline 200 & 30343 & $2 \cdot 711$ & 4 & $1 \cdot 356$ & 4 & 3.453 & 03018 \\
\hline 250 & 32025 & $3 \cdot 301$ & 4 & $1 \cdot 651$ & 4 & $3 \cdot 647$ & $0 \cdot 3020$ \\
\hline $30 \cdot 0$ & 33529 & $3 \cdot 858$ & 4 & 1.931 & 4 & $3 \cdot 825$ & 0.3024 \\
\hline 350 & 34804 & $4 \cdot 380$ & 4 & $2 \cdot 195$ & 4 & 4025 & 03031 \\
\hline $40 \cdot 0$ & 35963 & 4872 & 4 & $2 \cdot 445$ & 4 & $4 \cdot 217$ & $0 \cdot 3040$ \\
\hline $50 \cdot 0$ & 38104 & $5 \cdot 765$ & 4 & 2.908 & 4 & 4519 & 03071 \\
\hline $60 \cdot 0$ & 39780 & 6546 & 4 & $3 \cdot 322$ & 4 & 4.977 & 03109 \\
\hline 700 & 41194 & $7 \cdot 274$ & 4 & $3 \cdot 713$ & 4 & 5233 & 03146 \\
\hline 800 & 42535 & 7.965 & 4 & 4089 & 4 & $5 \cdot 431$ & 0.3183 \\
\hline 900 & 43716 & 8.632 & 4 & $4 \cdot 451$ & 4 & $5 \cdot 606$ & 0.3212 \\
\hline $100 \cdot 0$ & 44872 & $9 \cdot 312$ & 4 & $4 \cdot 821$ & 4 & 5539 & 03238 \\
\hline
\end{tabular}

a The power of 10 by which to multiply the first number. 
Table A2. Modfe P12

\begin{tabular}{|c|c|c|c|c|c|c|c|}
\hline \multirow{2}{*}{$\begin{array}{c}\tau \\
0000\end{array}$} & \multirow{2}{*}{$\frac{\begin{array}{c}T \\
\text { (degrees) }\end{array}}{10800}$} & \multicolumn{2}{|c|}{$\underset{\left(\text { dynes } / \mathrm{cm}^{2} \text { ) }\right.}{P_{g}}$} & \multicolumn{2}{|c|}{$\begin{array}{c}P_{e} \\
\left(\text { dynes } / \mathrm{cm}^{2}\right)\end{array}$} & \multirow{2}{*}{$\frac{\begin{array}{c}\overline{x+\sigma} \\
\left(\mathrm{cm}^{2} / \mathrm{g}\right)\end{array}}{0.700}$} & \multirow{2}{*}{$\begin{array}{c}\begin{array}{c}\sigma \\
\left(\mathrm{cm}^{2} / \mathrm{g}\right)\end{array} \\
0.2692\end{array}$} \\
\hline & & $1 \cdot 404$ & $1^{a}$ & $6 \cdot 622$ & $0^{\mathbf{a}}$ & & \\
\hline $0 \cdot 001$ & 11205 & $2 \cdot 559$ & 1 & $1 \cdot 207$ & 1 & 0849 & $0 \cdot 2694$ \\
\hline 0002 & 11475 & $3 \cdot 608$ & 1 & $1 \cdot 702$ & 1 & 0932 & $0 \cdot 2696$ \\
\hline 0.003 & 11715 & $4 \cdot 610$ & 1 & $2 \cdot 177$ & 1 & 0.974 & 0.2699 \\
\hline $0 \cdot 004$ & 11925 & $5 \cdot 612$ & 1 & $2 \cdot 651$ & 1 & 0.975 & $0 \cdot 2702$ \\
\hline 0005 & 12075 & $6 \cdot 535$ & 1 & 3-088 & 1 & 1.056 & 0.2704 \\
\hline 0.006 & 12195 & 7401 & 1 & $3 \cdot 499$ & 1 & 1123 & 0.2706 \\
\hline 0.007 & 12270 & $8 \cdot 237$ & 1 & $3 \cdot 895$ & 1 & 1161 & 02706 \\
\hline 0.008 & 12360 & 9039 & 1 & 4275 & 1 & 1211 & $0 \cdot 2708$ \\
\hline 0.009 & 12420 & $9 \cdot 809$ & 1 & 4640 & 1 & $1 \cdot 260$ & 02708 \\
\hline $0 \cdot 01$ & 12510 & $1 \cdot 056$ & 2 & $4 \cdot 997$ & 1 & $1 \cdot 291$ & $0 \cdot 2710$ \\
\hline 0.02 & 12930 & 1662 & 2 & $7 \cdot 875$ & 1 & 1586 & $0 \cdot 2718$ \\
\hline 003 & 13245 & $2 \cdot 213$ & 2 & $1 \cdot 050$ & 2 & $1 \cdot 736$ & 02726 \\
\hline 004 & 13530 & $2 \cdot 753$ & 2 & $1 \cdot 309$ & 2 & $1 \cdot 766$ & 02735 \\
\hline 005 & 13800 & $3 \cdot 282$ & 2 & 1564 & 2 & 1801 & $0 \cdot 2747$ \\
\hline 006 & 14025 & 3798 & 2 & $1 \cdot 814$ & 2 & $1 \cdot 844$ & 02758 \\
\hline 0.07 & 14205 & $4 \cdot 266$ & 2 & 2041 & 2 & 2021 & $0 \cdot 2768$ \\
\hline $0 \cdot 08$ & 14400 & $4 \cdot 734$ & 2 & $2 \cdot 270$ & 2 & $2 \cdot 021$ & $0 \cdot 2780$ \\
\hline 0.09 & 14550 & 5183 & 2 & $2 \cdot 490$ & 2 & 2096 & 02789 \\
\hline 0.10 & 14670 & 5616 & 2 & $2 \cdot 702$ & 2 & $2 \cdot 158$ & 02796 \\
\hline $0 \cdot 20$ & 15826 & $9 \cdot 578$ & 2 & 4676 & 2 & 2384 & $0 \cdot 2878$ \\
\hline $0 \cdot 30$ & 16650 & 1321 & 3 & $6 \cdot 508$ & 2 & $2 \cdot 569$ & 02928 \\
\hline 040 & 17310 & 1692 & 3 & $8 \cdot 375$ & 2 & 2512 & $0 \cdot 2957$ \\
\hline 050 & 17925 & $2 \cdot 069$ & 3 & 1027 & 3 & 2469 & 02976 \\
\hline $0 \cdot 60$ & 1849,5 & $2 \cdot 436$ & 3 & $1 \cdot 212$ & 3 & $2 \cdot 530$ & $0 \cdot 2988$ \\
\hline 0.70 & 19005 & 2803 & 3 & 1396 & 3 & $2 \cdot 528$ & 02996 \\
\hline 1.00 & 20415 & 3937 & 3 & 1965 & 3 & $2 \cdot 455$ & $0 \cdot 3008$ \\
\hline $1 \cdot 30$ & 21480 & 5060 & $\mathbf{3}$ & $2 \cdot 528$ & $\mathbf{3}$ & 2483 & 03012 \\
\hline 160 & 22440 & $6 \cdot 184$ & 3 & 3090 & 3 & 2488 & $0 \cdot 3014$ \\
\hline $1 \cdot 90$ & 23160 & $7 \cdot 281$ & 3 & $3 \cdot 639$ & 3 & $2 \cdot 554$ & $0 \cdot 3015$ \\
\hline $2 \cdot 20$ & 23805 & 8361 & $\mathbf{3}$ & 4179 & 3 & $2 \cdot 593$ & $0 \cdot 3015$ \\
\hline 250 & 24420 & 9420 & 3 & $4 \cdot 709$ & 3 & 2642 & 03016 \\
\hline 300 & 25335 & $1 \cdot 114$ & 4 & 5568 & 3 & 2706 & $0 \cdot 3016$ \\
\hline 350 & 26175 & $1 \cdot 283$ & 4 & 6412 & 3 & $2 \cdot 753$ & 03016 \\
\hline $4 \cdot 00$ & 26910 & $1 \cdot 447$ & 4 & 7234 & 3 & $2 \cdot 816$ & 03017 \\
\hline $4 \cdot 50$ & 27570 & $1 \cdot 608$ & 4 & 8041 & 3 & 2866 & 03017 \\
\hline $5 \cdot 00$ & 28245 & $1 \cdot 767$ & 4 & $8 \cdot 837$ & 3 & $2 \cdot 901$ & 03017 \\
\hline 550 & 28830 & 1.923 & 4 & 9617 & 3 & 2954 & $0 \cdot 3017$ \\
\hline 850 & 31890 & $2 \cdot 779$ & 4 & $1 \cdot 390$ & 4 & 3202 & $0 \cdot 3020$ \\
\hline $11 \cdot 50$ & 34200 & 3554 & 4 & 1.781 & 4 & $3 \cdot 476$ & 03029 \\
\hline $14 \cdot 50$ & 36255 & 4264 & 4 & $2 \cdot 143$ & 4 & $3 \cdot 779$ & 03048 \\
\hline $17 \cdot 50$ & 37920 & 4929 & 4 & 2488 & 4 & 4027 & 03074 \\
\hline $20 \cdot 50$ & 39450 & $5 \cdot 532$ & 4 & $2 \cdot 808$ & 4 & $4 \cdot 404$ & 03110 \\
\hline $23 \cdot 50$ & 41595 & $6 \cdot 121$ & 4 & $3 \cdot 137$ & 4 & $4 \cdot 50 \mathrm{I}$ & 03173 \\
\hline
\end{tabular}

a The power of 10 by which to multiply the first number. 
TABLE A3. ModiL P11

\begin{tabular}{|c|c|c|c|c|c|c|c|}
\hline \multirow{2}{*}{$\begin{array}{l}\tau \\
0000\end{array}$} & \multirow{2}{*}{$\frac{\begin{array}{c}T \\
\text { (degrees) }\end{array}}{14000}$} & \multicolumn{2}{|c|}{$\begin{array}{c}P_{\boldsymbol{g}} \\
\left(\text { dynes } / \mathrm{cm}^{2} \text { ) }\right.\end{array}$} & \multicolumn{2}{|c|}{$\begin{array}{c}P_{e} \\
\left(\text { dynes } / \mathrm{cm}^{2}\right)\end{array}$} & \multirow{2}{*}{$\begin{array}{c}\bar{c} \begin{array}{c}x+\sigma \\
\left(\mathrm{cm}^{2} / \mathrm{g}\right)\end{array} \\
0.4407\end{array}$} & \multirow{2}{*}{$\begin{array}{c}\begin{array}{c}\sigma \\
\left(\mathrm{cm}^{2} / \mathrm{g}\right)\end{array} \\
0.2980\end{array}$} \\
\hline & & $2 \cdot 181$ & $1^{\mathrm{a}}$ & $1 \cdot 084$ & $1^{\text {a }}$ & & \\
\hline 0.001 & 15040 & $4 \cdot 087$ & 1 & $2 \cdot 034$ & 1 & 0.5026 & $0 \cdot 2989$ \\
\hline 0.002 & 15400 & $5 \cdot 848$ & 1 & $2 \cdot 912$ & 1 & 0.5410 & 02993 \\
\hline 0.003 & 15720 & $7 \cdot 534$ & 1 & $3 \cdot 754$ & 1 & 05644 & 0.2996 \\
\hline 0.004 & 15940 & 9153 & 1 & $4 \cdot 562$ & 1 & 0.5861 & $0 \cdot 2998$ \\
\hline 0005 & 16140 & 1070 & 2 & $5 \cdot 334$ & 1 & 0.6025 & $0 \cdot 3000$ \\
\hline 0006 & 16300 & $1 \cdot 220$ & 2 & 6086 & 1 & 06274 & 0.3001 \\
\hline 0.007 & 16420 & 1363 & 2 & $6 \cdot 797$ & 1 & 0.6612 & $0 \cdot 3001$ \\
\hline 0008 & 16500 & 1.503 & 2 & 7496 & 1 & 06711 & $0 \cdot 3002$ \\
\hline 0.009 & 16600 & 1640 & 2 & $8 \cdot 177$ & 1 & 0.6891 & $0 \cdot 3002$ \\
\hline 0.01 & 16680 & $1 \cdot 772$ & 2 & $8 \cdot 840$ & 1 & 0.7061 & 0.3001 \\
\hline 002 & 17240 & $2 \cdot 906$ & 2 & $1 \cdot 450$ & 2 & $0 \cdot 8180$ & 0.3004 \\
\hline 003 & 17660 & $3 \cdot 944$ & 2 & 1.968 & 2 & 0.8852 & 0.3006 \\
\hline 0.04 & 18040 & $4 \cdot 925$ & 2 & $2 \cdot 458$ & 2 & 09291 & 0.3007 \\
\hline 0.05 & 18440 & $5 \cdot 853$ & 2 & 2.923 & 2 & 0.9726 & 0.3009 \\
\hline 0.06 & 18840 & 6.759 & 2 & $3 \cdot 376$ & 2 & 0.9936 & 0.3011 \\
\hline 0.07 & 19160 & $7 \cdot 645$ & 2 & 3.819 & 2 & 1.0110 & 0.3012 \\
\hline 0.08 & 19500 & $8 \cdot 526$ & 2 & $4 \cdot 260$ & 2 & 1.0155 & 0.3013 \\
\hline 0.09 & 19740 & $9 \cdot 392$ & 2 & $4 \cdot 693$ & 2 & 1.0319 & 0.3013 \\
\hline $0 \cdot 10$ & 20000 & $1 \cdot 025$ & 3 & $5 \cdot 122$ & 2 & $1 \cdot 0386$ & 0.3014 \\
\hline 0.20 & 21720 & $1 \cdot 787$ & 3 & 8931 & 2 & $1 \cdot 1591$ & 0.3016 \\
\hline $0 \cdot 30$ & 22800 & $2 \cdot 486$ & 3 & $1 \cdot 243$ & 3 & $1 \cdot 2549$ & 0.3016 \\
\hline $0 \cdot 40$ & 23700 & $3 \cdot 149$ & 3 & $1 \cdot 574$ & 3 & 1.3152 & $0 \cdot 3016$ \\
\hline 0.50 & 24520 & $3 \cdot 784$ & 3 & $1 \cdot 892$ & 3 & 1.3658 & 0.3017 \\
\hline 060 & 25260 & $4 \cdot 400$ & 3 & $2 \cdot 200$ & 3 & 14036 & $0 \cdot 3017$ \\
\hline $0 \cdot 70$ & 25900 & $5 \cdot 000$ & 3 & $2 \cdot 500$ & 3 & $1 \cdot 4383$ & $0 \cdot 3017$ \\
\hline 1.00 & 27760 & 6685 & $\mathbf{3}$ & $3 \cdot 343$ & 3 & 1.5037 & 0.3017 \\
\hline $1 \cdot 30$ & 29100 & $8 \cdot 287$ & 3 & $4 \cdot 144$ & 3 & 1.5820 & 03018 \\
\hline $1 \cdot 60$ & 30360 & $9 \cdot 824$ & 3 & 4.915 & 3 & $1 \cdot 6464$ & 03020 \\
\hline 1.90 & 31440 & $1 \cdot 130$ & 4 & $5 \cdot 653$ & 3 & $1 \cdot 7195$ & 0.3023 \\
\hline $2 \cdot 20$ & 32340 & $1 \cdot 270$ & 4 & $6 \cdot 361$ & 3 & $1 \cdot 7926$ & 03027 \\
\hline $2 \cdot 50$ & 33160 & $1 \cdot 405$ & 4 & 7040 & 3 & 1.8647 & 0.3033 \\
\hline 3.00 & 34400 & $1 \cdot 610$ & 4 & 8089 & 3 & $1 \cdot 9839$ & 0.3045 \\
\hline 3.50 & 35600 & 1.801 & 4 & $9 \cdot 073$ & 3 & $2 \cdot 1193$ & 0.3064 \\
\hline 4.00 & 36600 & 1.974 & 4 & 9.983 & 3 & $2 \cdot 2263$ & $0 \cdot 3086$ \\
\hline $4 \cdot 50$ & 37700 & $2 \cdot 147$ & 4 & 1.091 & 4 & $2 \cdot 2976$ & $0 \cdot 3116$ \\
\hline $5 \cdot 00$ & 38800 & $2 \cdot 305$ & 4 & $1 \cdot 177$ & 4 & $2 \cdot 4286$ & $0 \cdot 3150$ \\
\hline $5 \cdot 50$ & 39600 & $2 \cdot 458$ & 4 & $1 \cdot 260$ & 4 & $2 \cdot 5237$ & 0.3174 \\
\hline $8 \cdot 50$ & $\mathbf{4 2 8 8 0}$ & 3.358 & 4 & $1 \cdot 743$ & 4 & $2 \cdot 7339$ & 0.3254 \\
\hline 11.50 & 45500 & $4 \cdot 243$ & 4 & 2214 & 4 & $2 \cdot 6801$ & 0.3291 \\
\hline 1450 & 48120 & $5 \cdot 163$ & 4 & $2 \cdot 702$ & 4 & $2 \cdot 5696$ & 0.3311 \\
\hline 1750 & 50280 & $6 \cdot 156$ & 4 & $3 \cdot 225$ & 4 & $2 \cdot 4124$ & 0.3320 \\
\hline 2050 & 52280 & $7 \cdot 246$ & 4 & $3 \cdot 799$ & 4 & $2 \cdot 2333$ & 0.3325 \\
\hline $23 \cdot 50$ & 55140 & $8 \cdot 556$ & 4 & $4 \cdot 488$ & 4 & 1.9187 & 0.3329 \\
\hline
\end{tabular}

a The power of $10 \mathrm{by}$ which to multiply the first number. 
Table A4. Moder P8

\begin{tabular}{|c|c|c|c|c|c|c|c|}
\hline \multirow{2}{*}{$\frac{\tau}{0000}$} & \multirow{2}{*}{$\frac{\begin{array}{c}T \\
\text { (degrees) }\end{array}}{18900}$} & \multicolumn{2}{|c|}{$\underset{\left(\text { dynes } / \mathrm{cm}^{2}\right)}{P_{g}}$} & \multicolumn{2}{|c|}{$\begin{array}{c}P_{e} \\
\left(\text { dynes } / \mathrm{cm}^{2}\right)\end{array}$} & \multirow{2}{*}{$\frac{\begin{array}{c}\overline{x+\sigma} \\
\left(\mathrm{cm}^{2} / \mathrm{g}\right)\end{array}}{03530}$} & \multirow{2}{*}{$\begin{array}{c}\begin{array}{c}\sigma \\
\left(\mathrm{cm}^{2} / \mathrm{g}\right)\end{array} \\
0.3017\end{array}$} \\
\hline & & $2 \cdot 547$ & $1^{a}$ & $1 \cdot 274$ & $1^{\mathrm{a}}$ & & \\
\hline 0.001 & 19732 & 4903 & 1 & $2 \cdot 452$ & 1 & 03786 & 0.3017 \\
\hline 0.002 & 20336 & $7 \cdot 136$ & 1 & $3 \cdot 568$ & 1 & 0.3972 & $0 \cdot 3017$ \\
\hline 0.003 & 20765 & $9 \cdot 276$ & 1 & 4638 & 1 & 0.4123 & $0 \cdot 3017$ \\
\hline 0004 & 21118 & $1 \cdot 135$ & 2 & $5 \cdot 673$ & 1 & $0 \cdot 4247$ & $0 \cdot 3017$ \\
\hline 0005 & 21395 & $1 \cdot 336$ & 2 & $6 \cdot 678$ & 1 & 04354 & $0 \cdot 3017$ \\
\hline 0.006 & 21622 & $1 \cdot 532$ & 2 & $7 \cdot 659$ & 1 & 0.4452 & 03017 \\
\hline 0007 & 21823 & $1 \cdot 724$ & 2 & $8 \cdot 618$ & 1 & 0.4538 & 03017 \\
\hline 0008 & 21974 & 1.912 & 2 & 9.558 & 1 & 0.4622 & 03017 \\
\hline 0.009 & 22100 & 2095 & 2 & $1 \cdot 048$ & 2 & 0.4708 & 03017 \\
\hline 001 & 22226 & $2 \cdot 276$ & 2 & $1 \cdot 138$ & 2 & 04788 & $0 \cdot 3017$ \\
\hline 0.02 & 22882 & $3 \cdot 840$ & 2 & 1.920 & 2 & 0.5402 & 03017 \\
\hline $0 \cdot 03$ & 23512 & $5 \cdot 283$ & 2 & 2641 & 2 & $0 \cdot 5802$ & 03017 \\
\hline 004 & 23990 & 6638 & 2 & $3 \cdot 319$ & 2 & $0 \cdot 6121$ & $0 \cdot 3017$ \\
\hline 0.05 & 24419 & $7 \cdot 923$ & 2 & 3962 & 2 & 06403 & $0 \cdot 3017$ \\
\hline 0.06 & 24822 & $9 \cdot 159$ & 2 & 4.580 & 2 & $0 \cdot 6637$ & 03017 \\
\hline 007 & 25124 & 1.035 & 3 & $5 \cdot 175$ & 2 & 06854 & 03017 \\
\hline 0.08 & 25376 & $1 \cdot 150$ & $\mathbf{3}$ & $5 \cdot 749$ & 2 & 07061 & 03017 \\
\hline 0.09 & 25654 & $1 \cdot 261$ & 3 & $6 \cdot 306$ & 2 & 07224 & 03017 \\
\hline 010 & 25956 & $1 \cdot 370$ & 3 & $6 \cdot 851$ & 2 & 0.7360 & 0.3018 \\
\hline $0 \cdot 20$ & 27972 & $2 \cdot 307$ & 3 & $1 \cdot 154$ & 3 & 0.8429 & 0.3019 \\
\hline $0 \cdot 30$ & 29358 & $3 \cdot 151$ & 3 & $1 \cdot 577$ & $\mathbf{3}$ & 09238 & 03022 \\
\hline $0 \cdot 40$ & 30492 & 3.929 & 3 & 1967 & 3 & $0 \cdot 9906$ & $0 \cdot 3026$ \\
\hline 050 & 31424 & $4 \cdot 651$ & 3 & $2 \cdot 331$ & 3 & 1.0507 & 03031 \\
\hline 0.60 & 32306 & 5327 & 3 & 2673 & 3 & $1 \cdot 1052$ & 03040 \\
\hline 070 & 33138 & 5.967 & 3 & $3 \cdot 000$ & 3 & $1 \cdot 1581$ & 0.3051 \\
\hline 100 & 35028 & $7 \cdot 623$ & 3 & 3857 & $\mathbf{3}$ & $1 \cdot 3192$ & 03089 \\
\hline 130 & 36414 & $9 \cdot 084$ & 3 & $4 \cdot 624$ & $\mathbf{3}$ & 14471 & 03128 \\
\hline $1 \cdot 60$ & 37750 & 1046 & 4 & 5361 & 3 & 1.5211 & 03171 \\
\hline 1.90 & 38909 & 1178 & 4 & 6068 & 3 & 16196 & 0.3206 \\
\hline $2 \cdot 20$ & 39841 & 1305 & 4 & $6 \cdot 746$ & $\mathbf{3}$ & $1 \cdot 6782$ & 0.3231 \\
\hline 250 & 40622 & 1.429 & 4 & $7 \cdot 409$ & 3 & $1 \cdot 7067$ & $0 \cdot 3248$ \\
\hline 300 & 41832 & $1 \cdot 633$ & 4 & $8 \cdot 491$ & 3 & $1 \cdot 7065$ & 0.3270 \\
\hline 350 & 43168 & 1. 832 & 4 & 9557 & 3 & 17131 & 03289 \\
\hline 400 & 44302 & 2036 & 4 & 1064 & 4 & 16912 & 03301 \\
\hline $4 \cdot 50$ & 45335 & 2246 & 4 & 1175 & 4 & 16566 & 03309 \\
\hline 500 & 46368 & 2458 & 4 & 1287 & 4 & 16349 & 0.3315 \\
\hline 550 & 47326 & 2670 & 4 & 1398 & 4 & 16404 & 0.3319 \\
\hline 850 & 51962 & 4181 & 4 & 2193 & 4 & 15245 & $0 \mathbf{3 3 2 9}$ \\
\hline $11 \cdot 50$ & 54810 & $5 \cdot 776$ & 4 & 3.030 & 4 & $1 \cdot 4848$ & 0.3331 \\
\hline $14 \cdot 50$ & 57305 & $7 \cdot 436$ & 4 & 3.903 & 4 & $1 \cdot 4376$ & 03332 \\
\hline 1750 & 59522 & 9070 & 4 & $4 \cdot 760$ & 4 & 1.4402 & 03333 \\
\hline $20 \cdot 50$ & 61715 & $1 \cdot 071$ & 5 & $5 \cdot 623$ & 4 & 14110 & 0.3333 \\
\hline 2350 & 65218 & 1253 & 5 & 6.576 & 4 & 12833 & $0 \cdot 3334$ \\
\hline
\end{tabular}

a The power of 10 by wheh to multiply the first number. 


\section{REFERENCES}

ALLER, L. H. (1949) Ap. J. 109, 244.

ALLER, L. H. (1961) The Abundances of the Elements, Interscience, New York, p. 192.

ALLER, L. H. (1966) Unpublished lust privately communicated.

Aller, L. H. and JUGAKo, J. (1958) Ap. J. Suppl. 4, 109.

AVRETT, E. H. and Krook, M. (1963) J. Quant Spectrosc. Radiat. Transf. 3, 107.

Bernacon, P. L. (1968) Atti del XI Convegno S.A.I

Canion, A. J. and Pickerina, E. C. (1918) Ann. Harv Coll. Obs., vols. 91-99

Colurns, G. W. (1966) Ap. J. 146, 914.

Fadlener, J., Roxburgh, I. W., and Strittmatter, P. A (1968) Ap. J. 151, 203.

Garrison, R. F (1967) Ap.J. 147, 1003.

GRIEM, H. R. (1960) Ap. J. 132, 883.

De Groot, M. and UnderHILL, A. B. (1964) Bull. Astr. Inst. Netherlands 17, 280.

GrygaR, J. (1964) Bull. Astr. Inst. Netherlands 17, 280.

GUTJAdume, C. (1966) Bull. Astr. Inst Netherlands 18, 175.

Hanbury Brown, R , Davis, J., Allen, L. R. and Rome, J. (1967) M.N.R.A.S 137, 393

HARDORP, J. (1966) Zs. Ap. 63, 137.

Hriñze, J. R. W. (1968) Bull. Astr. Inst. Netherlands, $20,1$.

HeIntze, J. R. W. (1969) Proc. Therd Harvard-Smithsonian Conference Theory and Observation of Normal Stellar Atmospheres, edited by O. Gingerıch, M I. T. Press, Cambridge, Mass., p. 265.

Van Helden, R. (1966) Bull. Astr. Inst. Netherlands, 18, 367.

JUGAKO, J. and SARGENT, W. L. W. (1968) Ap. J. 151, 259.

Jugaku, J., Sargent, W. L. W. and Greenstein, J. L. (1961) Ap J. 134, 783.

Katzofen, W. and Strom, S. E. (1966) J. Quant. Spectrosc. Radiat. Transf. 6, 653.

Ledodx, P. and Renson, P. (1966) Ann. Rev. Astr. Ap. 4, 293.

MoWhirter, R. W. P. (1965) Plasma Diagnostıc Techniques, edited by Huddlestone and Leonard, Academic Press, London, p. 205.

MrHaLAS, D. (1965) Ap J. Suppl. 9, 321, (No. 92).

Mrhalas, D. (1967) Ap. J.149, 169.

MrHaLAs, D. and Stone, D. E. (1968) Ap. J. 151, 293.

Mruse, E. A. (1930) Hb. Ap. 3, Part 1, 68; reprinted in Selected Papers on the Transfer of Radiation, edited by D. H. Menzel, Dover Publ, New York, 1966, p. 77.

Morgan, W. W and JoHnson, H. L. (1953) $A p$. J. 117, 313.

Moraan, W. W. and Kennax, P. C. (1951) Astrophysics, edited by Hynek, MeGraw-Hill, New York, p. 12

MoRTon, D. C., (1965) $A p . J .141,73$.

Packer, J. C. (1965) Ann. Rev. Astr. Ap. 3, 135.

Petrie, R. M. (1964) Pub. Dominion Ap Obs 12, 317.

Plaskett, J. S and Pearce, J. A. (1930) Publ. Dom Astrophys. Obs. $5,1$.

Roberts, J. R. and EckerLe, K. L. (1967) Phys. Rev. 158, 87.

Sargent, W. L. W. (1964) Ann Rev. Astr Ap 2, 297.

Scholz, M. T. (1965) Astr. Ab. Hamburger Sternw. 7, No. 6

Scholz, M. T. (1967) Zs. Ap. 65, 1.

Sмттн, M. (1967) Ap. J. 147, 158.

Strom, S. E. and AVRETT, E. H. (1965) Ap. J. Suppl. 12, 1, (No. 103).

Strom, S. E. and KaLkofen, W. (1966) $A p . J$ 144, 76

Streve, O. and EuveY, C. T. (1934) $A p . J$ 79, 409.

UNDERHILL, A. B. (1948) Ap. J. 107, 349.

UNDERHILL, A. B. (1957) $A p . J .126,28$.

Underhtll, A. B (1962a) Publ Dom Ap. Obs. 11, No. 23

UNDERHILl, A. B. (1962 b) Publ. Dom. Ap. Obs. 11, No. 24.

UNDERHIL, A B. (1963) Bull Astr. Inst. Netherlands 17, 161.

Undermill, A B. (1966 a) Vistas in Astronomy, edited by A Beer 8, p. 41; Pergamon Press, London.

UndernmL, A. B. (1966 b) The Early-Type Stars, D. Reidel, Dordrecht, p. 167.

UNDERHILL, A. B. (1967) Bull. Astr. Inst. Netherlands, 19, 173.

UNDERHILL, A. B. (1968a) Bull. Astr. Inst Netherlands, 19, 500.

UNDERHTLC, A. B. (1968b) Ap. J. 151, 765.

UNDERHILL, A B. (1968c) Bull Astr Inst. Netherlands 19, 526.

Underhmu, A. B. (1968d) Bull Astr. Inst. Netherlands, $19,537$. 
Undermill, A B (1969) Theory and Observation of Normal Stellar Atmospheres, edited by O. Gingerıch, M. I. T. Press, Cambridge, Mass., p. 207.

UnderhiLl, A. B. and De Groot, M. (1964) Bull. Astr. Inst. Netherlands, 17, 453.

UnderhtLL, A. B. and DE Groot, M. (1965) Rech Astr. Obs. Utrecht, 17, No 3.

UNDERHWL, A. B. and WALKER, G. A. H (1966) M.N.R.A S. 131, 475.

Unsold, A. (1955) Physnk der Sternatmospharen, Springer Verlag, Berlın, p. 508.

Wright, K. O., Lew, E. K, JAcobsen, T. V. and Grennstein, J L. (1963) Publ. Dom. Astr. Obs.12, No. 7. 